\title{
Discovering beautiful attributes for aesthetic image analysis
}

\author{
Luca Marchesotti · Naila Murray · Florent Perronnin
}

Received: date / Accepted: date

\begin{abstract}
Aesthetic image analysis is the study and assessment of the aesthetic properties of images. Current computational approaches to aesthetic image analysis either provide accurate or interpretable results. To obtain both accuracy and interpretability by humans, we advocate the use of learned and nameable visual attributes as mid-level features. For this purpose, we propose to discover and learn the visual appearance of attributes automatically, using a recently introduced database, called AVA, which contains more than 250,000 images together with their aesthetic scores and textual comments given by photography enthusiasts. We provide a detailed analysis of these annotations as well as the context in which they were given. We then describe how these three key components of AVA - images, scores, and comments - can be effectively leveraged to learn visual attributes. Lastly, we show that these learned attributes can be successfully used in three applications: aesthetic quality prediction, image tagging and retrieval.
\end{abstract}

Keywords image aesthetics - database · visual attributes $\cdot$ textual attributes

Luca Marchesotti

Beautifeye

Suite 203, Media Cube, IADT

Baile Átha Cliath, Ireland

Xerox Research Centre Europe

Meylan, France

E-mail: luca@beautifeye.co

Naila Murray and Florent Perronnin

Xerox Research Centre Europe

Meylan, France

E-mail: Firstname.Lastname@xrce.xerox.com

\section{Introduction}

The volume of visual data we handle on a daily basis is growing exponentially, and will continue to do so due to the availability of ubiquitous and cheap sensors, sharing platforms and new social trends. Artificial intelligence systems have proven useful for processing and interpreting this preponderance of data. In the last decade, the computer vision and image retrieval community was focused on developing tools for semantic analysis of multimedia content. While this is still a very active research field, new questions are arising. These questions are about visual properties beyond visual semantics, such as image preference (Datta et al 2006), affectiveness (Machajdik and Hanbury 2010), and memorability (Isola et al 2011), as well as object importance (Berg et al 2012). Answering subjective, humancentric questions such as "would someone find this image aesthetically pleasing" is very challenging, even for humans. However, it was experimentally shown that these visual cognition phenomena can be predicted using data-driven approaches (Luo and Tang 2008; Datta et al 2008; Machajdik and Hanbury 2010; Dhar et al 2011; Marchesotti et al 2011; Murray et al 2012a). In this work we focus on image preference: that is, whether people will like an image and which visual elements makes it un/attractive.

Early work on image preference prediction Datta et al 2006, Ke et al 2006) proposed to mimic the best practices of professional photographers. In a nutshell, the idea was (i) to select rules (e.g. "contains opposing colors") from photographic resources such as (Kodak 1982 ) and (ii) to design for each rule a visual feature to predict the image compliance (e.g. a color histogram). Many subsequent works have focused on adding new photographic rules and on improving the visual fea- 
tures of existing rules (Luo and Tang 2008; Dhar et al 2011). As noted for instance in (Dhar et al 2011) these rules can be understood as visual attributes (Ferrari and Zisserman 2007, Lampert et al 2009; Farhadi et al 2009 ), i.e. medium-level descriptions whose purpose is to bridge the gap between the high-level concepts to be recognized (beautiful vs. ugly in our case) and the lowlevel pixels. However, there are at least two issues with such an approach to aesthetic prediction. Firstly, the hand-selection of attributes from a photographic guide is not exhaustive and does not give any indication of when, and to what extent, such rules are used. Secondly, hand-designed visual features only imperfectly model the corresponding rules.

As an alternative to rules and hand-designed features, it was proposed in (Marchesotti et al 2011) to rely on generic features such as the GIST (Oliva and Torralba 2001), the bag-of-visual-words (BOV) (Csurka et al 2004) or the Fisher vector (FV) (Perronnin et al 2010). While it was shown experimentally that such an approach can lead to improved results with respect to hand-designed attribute techniques, a major shortcoming is that interpretability of the results is lost. In other words, while it is possible to say that an image has a high or low aesthetic value, it is impossible to tell why. We thus raise the following question: can we preserve the advantages of generic features and obtain interpretable results? In this work, we will address this problem by discovering and learning attributes automatically.

As described by (Parikh and Grauman 2011b), "[a]ttributes represent a class-discriminative, but not class-specific property that both computers and humans can decide on". Such a statement implies that attributes should be understandable by humans. Because selecting attributes by hand-picking photographic rules is problematic, we intend to automatically discover attributes using a data-driven approach. A natural way to enforce interpretability of the automatically discovered attributes is to mine them from natural text corpora, as done for instance in (Berg et al 2010). We adopt this approach, and mine attributes using aestheticsrelated textual terms associated with images. The discovery process is as follows: (i) textual image meta-data are used to form a vocabulary of aesthetic terms; (ii) the discriminability of each vocabulary term is assessed and the most discriminative terms are retained as textual attributes; (iii) visual appearance models for these textual attributes are trained using generic image descriptors and the most detectable models are retained as visual attributes.

Such an approach however, has a key requirement: a database with a unique conjunction of aesthetics- related content, namely (i) textual meta-data from which to mine for aesthetic terms; (ii) aesthetic preference scores to provide supervisory information when assessing the discriminability of attributes; (iii) images on which to train visual attribute models for textual aesthetic attributes. While several datasets exist which contain images and associated preference scores, to our knowledge only the recently-introduced AVA dataset (Murray et al 2012a) contains the full set of required content. AVA contains more than 250,000 images along with preference score distributions and textual comments given to images by photography enthusiasts. As such, we propose to leverage AVA as an essential resource for our approach.

The main contributions of our proposed method are thus the following:

1. An in-depth analysis of the AVA dataset, and in particular its textual comments and aesthetic preference scores.

2. A novel approach to aesthetic image analysis which combines the benefits of "attribute-based" and "generic" techniques by (i) automatically discovering discriminative textual attributes using user comments and preference scores (step 1 in Fig. 11; and (ii) supervised learning of detectable visual attributes using textual attributes and generic visual features (step 2 in Fig. 11).

3. The application of the learned visual attributes to three different scenarios: aesthetic quality prediction, image classification and retrieval (step 3 in Fig. 11.

The remainder of this work is organized as follows: in section 2 we review works related to aesthetic analysis and attributes. We then introduce the AVA dataset and explain why it is an essential resource for aesthetic attribute learning (section 3). In section 3.3 we analyze aesthetic preference as expressed by real-valued scores while in section 3.4 we analyze aesthetic preference as expressed in textual comments. We then introduce the proposed approach to discover attributes that consists of (i) mining for discriminative textual attributes using the user comments and user scores (section 4) and (ii) learning visual attributes by modeling the visual appearance of textual attributes using generic visual features (section 5). In section 6, we show practical applications of these attributes.

This paper extends our previous work (Murray et al 2012a (Marchesotti and Perronnin 2013) with 1) a more detailed quantitative and qualitative analysis of the textual comments included in the AVA corpus, 2) an expanded quantitative evaluation of the textual features derived from these comments, 3) a quantitative assessment of the generalization performance of our learned 


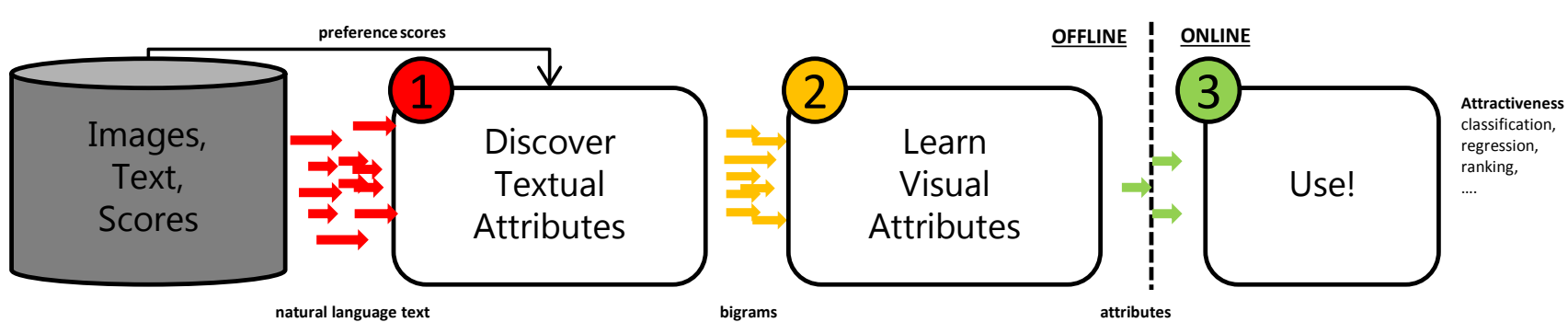

Fig. 1 We propose an end-to-end pipeline capable of learning visual attributes using information contained in the AVA dataset. Textual attributes are automatically discovered using textual comments and preference scores. Visual attribute models for these textual attributes are then learned using generic features extracted from images, as well as preference scores. See sections 4 and 5 for further details.

visual attributes on a different image corpus and 4) an expanded image retrieval application to include joint attribute-semantic queries.

\section{Related work}

The study of aesthetics spans millennia, from the works of philosophers such Plato to those of researchers today in fields as diverse as neuroscience, psychology, and computer science (Shelley 2012b; Leder et al 2004; Chatterjee 2011). This highly inter-disciplinary interest in the topic is a natural outcome of the complex and multi-faceted nature of aesthetics, which is defined in the American Heritage ${ }^{\circledR}$ Dictionary of the English Language ("aesthetics" 2012) as "the study of the mind and emotions in relation to the sense of beauty."

One major debate in the aesthetics research community surrounds the relative influence of subjective versus objective factors in aesthetic appreciation (Shelley 2012b). This debate has been ongoing at least since Baumgarten argued that aesthetic appreciation was the result of objective reasoning (Hammermeister 2002), while David Hume and Edmund Burke(Gracyk 2011; Shelley 2012a) took the opposing view that aesthetic appreciation was due to induced feelings.

For photography, which is the subject of our work, there are generally-accepted principles and techniques that are used by artists themselves to enhance the aesthetic quality of their artworks (Krages 2005). Examples include the "rule of thirds" compositional rule and "color harmony" guidelines (Krages 2005; Jacobson and Ostwald 1948). Note that these and other guidelines may be applicable to other pictorial art-forms such as paintings, which nonetheless remain out of the scope of this work.

These principles and techniques may have arisen due to both objective and subjective/cultural factors. However, what is critical for data-driven image aesthetics analysis is that they are often detectable using machine learning techniques and training data. The computer vision community has used detectable principles and techniques in order to design systems that attempt to predict the average response of an observer when asked questions such as "do you find this image aesthetically pleasing?", or "how would you rate this image on a score of 1 to 10 ?".

As this work discovers attributes relevant for image aesthetics analsyis, we review the literature on aesthetics prediction and attributes.

Computational image aesthetics prediction: As mentioned above, the computer vision community has in recent years developed data-driven approaches for analyzing pictorial artworks, particularly paintings and photographs. Such approaches use standard machine learning techniques such as linear classifiers or regressors to predict aesthetic annotations. Therefore the bulk of research effort has focused on designing appropriate visual features for representing image aesthetic characteristics. In general, these features attempt to capture specific aesthetic principles and techniques related to composition and the use of color and light (Datta et al |2006, Ke et al 2006, Luo and Tang 2008, Obrador et al 2010; Dhar et al 2011; Luo et al 2011. \begin{tabular}{|l|l|l|l|}
\hline Joshi et al 2011; San Pedro et al 2012, Obrador et al & 20
\end{tabular} 2012 ).

Datta's seminal work on aesthetic prediction extracted 56 visual features from an image and used these to train a statistical model to automatically classify an image as being of "beautiful" or "ugly" aesthetic quality (Datta et al 2006). The features included relative color frequencies, mean pixel intensity, mean pixel saturation and mean pixel hue. Photographic rules of thumb such as the rule-of-thirds were also incorporated as well as other features related to texture, aspect ratio, and low depth-of-field.

There have been many other works in this line, such as that of Ke et al. (Ke et al 2006) who proposed features capturing the spatial distribution of edges, color, blur, and brightness. Luo \& Tang (Luo and Tang 2008) extracted semantic features describing lighting, color, 
and composition from the foreground image region after segmentation. Dhar et al. (Dhar et al 2011) proposed the use of human-describable attributes related to composition, illumination and the image content. In (Li et al 2010), face-specific aesthetic features such as individual face expressions, individual face poses, and between-face distances were captured and used to assess and improve portraiture.

As mentioned before, it is difficult to define an exhaustive list of aesthetics-relevant image descriptors. An alternative approach, proposed by (Marchesotti et al 2011) is to use general-purpose image signatures to train aesthetics models. In this work the Bag-OfVisual-words descriptor (Csurka et al 2004, Sivic and Zisserman 2003) and the Fisher Vector (FV, (Perronnin and Dance 2007; Perronnin et al 2010)), based on SIFT (Lowe 1999) and color statistics features, were shown to achieve state-of-the-art aesthetic classification results. The authors posited that generic features are able to implicitly encode the aesthetic properties of an image. In addition, the spatial pyramid framework (Lazebnik et al 2006) was able to roughly encode compositional information. Some recent works have complemented visual features with aethetic features mined from textual data (San Pedro et al 2012; Geng et al 2011), by generating word frequency or TFIDF vectors from comments given to images by individuals.

The promising results obtained by various aesthetics models have enabled the development of prototypes for not only assessing but also improving image aesthetics (Joshi et al 2011). In particular, the web application ACQUINE (Datta and Wang 2010) allows one to upload images and receive a real-valued aesthetic score. Another such system, OSCAR (Yao et al 2012), is a mobile application which provides on-line feedback to assist the user in improving an image's composition or colorfulness.

Visual and textual attributes: There is a significant body of work on attribute learning in the computer vision and multimedia literature. This is a costeffective alternative to hand-listing attributes (Ferrari and Zisserman 2007; Lampert et al 2009) and to architectures which require a human in the loop (Parikh and Grauman 2011a). Existing solutions (Berg et al 2010, Wang et al 2009: Yanai and Barnard 2005) were typically developed for visual object recognition tasks. Wang et al (2009) proposes to mine pre-existing natural language resources. Berg et al (2010) uses mutual information to learn attributes relevant for e-commerce categories (handbags, shoes, earrings and ties). Duan et al (2012) uses latent CRF to discover detectable and discriminative attributes. Donahue and Grauman (2011) learned models for pre-determined nameable visual attributes and applied them in scene and human attractiveness classification tasks. Moreover, approaches such as (Rohrbach et al 2010) use natural language text in the form of captions or surrounding image text. Only Orendovici and Wang (2010) take into account text to devise aesthetic attributes, but the process is entirely manual.

In contrast to the reviewed works which hand-pick aesthetic attributes, we aim to automatically discover them from textual data, with preference scores as supervisory information. We next describe the dataset, AVA, that we will leverage for training our models.

\section{AVA: A large-scale database for aesthetic visual analysis}

AVA (Aesthetic Visual Analysis) is a publicly available database for aesthetics analysis which we recently introduced in (Murray et al 2012a). In what follows, we first compare AVA to related databases, and describe their limitations for our goal of automatic discovery of mid-level image representations for aesthetic analysis. We then provide a detailed analysis of AVA, focusing on 3 key components: (i) its images; (ii) its real-valued score annotations; and (iii) its textual comments.

\subsection{AVA and Related Databases}

In addition to AVA, there exist several public image databases in current use which contain aesthetic annotations. In this section, we compare the properties of these databases to those of AVA and discuss the features that differentiate AVA from such databases. A summary of this comparison is shown in Table 1.

Photo.net, PN (Datta et al 2006): PN contains 3,581 images from the social network Photo.net. In this online community, members are instructed to give two scores from 1 to 7 for an image. One score corresponds to the image's aesthetics and the other to the image's originality. The dataset includes the mean aesthetic score and the mean originality score for each image. As described in (Datta et al 2006), the aesthetic and originality scores are highly correlated, with little disparity between these two scores for a given image. This is probably due to the difficulty of separating these two characteristics of an image. As the two scores are therefore virtually interchangeable, works using PN have restricted their analysis to the aesthetic scores. Figure 2 shows sample photos of high quality with their scores and number of votes. 


\begin{tabular}{|l|c|c|c|c|c|}
\hline & AVA & PN & CUHK & CUHKPQ & CLEF \\
\hline \hline Large scale & Y & N & N & N & Y \\
\hline Score distr. & Y & Y & N & N & N \\
\hline $\begin{array}{l}\text { Rich } \\
\text { annotations }\end{array}$ & $\mathrm{Y}$ & N & Y & Y & Y \\
\hline Semantic labels & Y & N & N & Y & Y \\
\hline Style labels & Y & N & N & N & Y \\
\hline
\end{tabular}

Table 1 Comparison of the properties of current databases containing aesthetic annotations. AVA is large-scale and contains score distributions, rich annotations, and semantic and style labels.
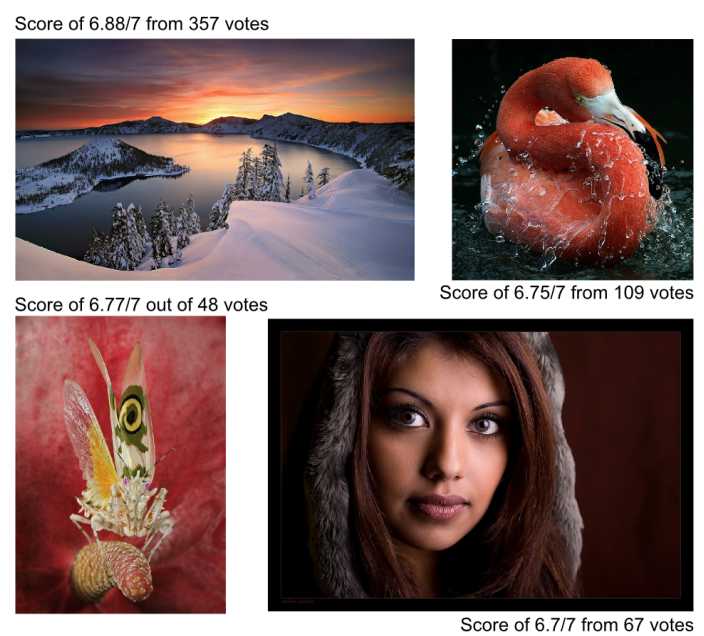

Fig. 2 Photos highly rated by peer voting in an on-line photo sharing community (photo.net).
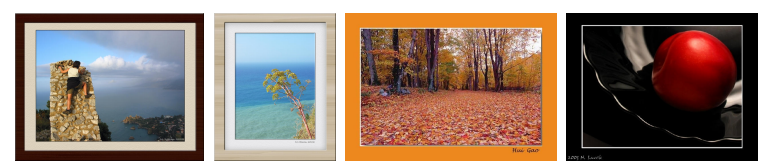

Fig. 3 Sample images from PN with borders manually created by photographers to enhance the photo visual appearance.

Upon visual inspection of $\mathrm{PN}$, we have noticed a correlation between images receiving a high grade and the presence of frames manually created by the owners to enhance the visual appearance (see examples in Figure 3). In fact, we manually detected that more than $30 \%$ of the images are framed.

In addition to this bias, many images in $\mathrm{PN}$ have been scored by very few users. In fact, the images were included on the condition that they had received scores from at least two users. In contrast, each image included in AVA has at least 78 votes. In addition, AVA contains approximately 70 times as many images.

CUHK (Ke et al 2006): CUHK contains 12,000 images, half of which are considered high quality and the rest labeled as low quality. (Ke et al 2006) observed the same bias for images with border as we did for PN, so they removed all the frames from the images they released. The images were obtained by retaining the top and bottom $10 \%$ (in terms of mean scores) of 60,000 images randomly crawled from www.dpchallenge.com. Our dataset differs from CUHK in several ways. While AVA includes more ambiguous images, CUHK only contains images with a very clear consensus on their score. As a consequence, the images in CUHK are much less representative of the range of images, in terms of aesthetic quality, that one would find in a real-world application such as re-ranking images returned by a search on the web. In addition, CUHK is no longer a challenging dataset for classification; recent methods achieved accuracies superior to $90 \%$ on this dataset (Marchesotti et al 2011). Finally, CUHK provides only binary labels ( $1=$ high quality images, $0=$ low quality images) whereas AVA provides an entire distribution of scores for each image.

CUHKPQ (Luo et al 2011): CUHKPQ consists of 17,690 images obtained from a variety of on-line communities and divided into 7 semantic categories. Each image was labeled as either high or low quality by at least 8 out of 10 independent viewers. Therefore this dataset consists of very high consensus images and their binary labels. Like CUHK, it is not a challenging dataset for the problem of binary classification: the method of (Luo et al 2011) obtained Area under the ROC curve (AROC) values between 0.89 and 0.95 for all semantic categories. Also like CUHK, the images in the dataset do not span the full range of images, in terms of aesthetic quality, that one is likely to find in a real-world aesthetic prediction application. In addition, despite the fact that AVA shares similar semantic annotations, it differs in terms of scale and also in terms of consistency. In fact, CUHKPQ was created by mixing high quality images derived from photographic communities and low quality images provided by university students.

MIRFLICKR/Image CLEF: Visual Concept Detection and Annotation Task 2011 (Müller et al 2010): MIRFLICKR is a large dataset introduced in the community of multimedia retrieval. It contains 1 million images collected from Flickr, along with textual tags, aesthetic annotations (Flickr's interestingness flag) and EXIF meta-data. A sub-part of MIRFLICKR was used by CLEF (the Cross-Language Evaluation Forum) to organize two challenges on "Visual Concept Detection". For these challenges, the basic annotations were enriched with emotional annotations and with some tags related to photographic style. It is probably the dataset closest to AVA but it lacks rich aesthetic preference annotations. In fact, only the "interestingness" flag is available to describe aesthetic preference. Some of the 44 visual concepts available might be re- 
lated to AVA photographic styles but they focus on two very specific aspects: exposure and blur. Only the following categories are available: neutral illumination, over-exposed, under-exposed, motion blur, no blur, out of focus, and partially blurred. In addition, the number of images with such style annotations is limited.

\subsection{AVA and its annotations}

AVA contains photographic images and a rich variety of associated meta-data, derived from www.dpchallenge .com. To our knowledge, AVA represents the first attempt to create a large database containing a unique combination of heterogeneous annotations. The peculiarity of this database is that it is derived from a community where images are uploaded and scored in response to photographic challenges. Each challenge is defined by a title and a short description (see Fig. 4 for a sample challenge).

Using this interesting characteristic, we associated each image in AVA with the information of its corresponding challenge. This information can be exploited in combination with aesthetic scores or semantic tags to gain an understanding of the context in which such annotations were provided. We created AVA by collecting approximately 255,000 images covering a wide variety of subjects on 1,447 challenges. We combined the challenges with identical titles and descriptions and we reduced them to 963 . Each image is associated with a single challenge.

In AVA we provide three types of annotations:

Aesthetic annotations: Each image is associated with a distribution of scores which correspond to individual votes. The number of votes per image ranges from 78 to 549, with an average of 210 votes. Such score distributions represent a gold mine of aesthetic judgments generated by hundreds of amateur and professional photographers with a practiced eye. In addition, AVA contains rich textual comments given to users by other community members. We believe that such annotations have a high intrinsic value because they capture the way hobbyists and professionals understand visual aesthetics.

Semantic annotations: We provide 66 textual tags describing the semantics of the images. Approximately 200,000 images contain at least one tag, and 150,000 images contain 2 tags. The frequency of the most common tags in the database can be observed in Fig. 5 .

Photographic style annotations: Despite the lack of a formal definition, we understand photographic style as a consistent manner of shooting photographs
TITLE: Skyscape

Description:

Make the sky the subject

of your photo this week.

Stats

Voting Dates:
13/07/2010 - 19/07/2010

Numbers \& Statistics:

Submissions: 136
Disqualifications: 1

Disqualifications: 1
Votes: 16,009

Comments: 595

Average Score: 5.64014
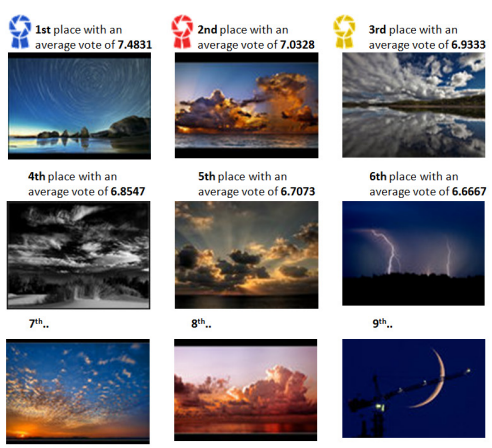

$8^{\text {th. }}$.

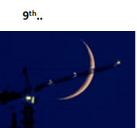

Fig. 4 A sample challenge entitled "Skyscape" from the social network www.dpchallenge. com. Users submit images that should conform to the challenge description and be of high aesthetic quality. The submitted images are rated by members of the social network during a finite score period. After this period, the images are ranked by their average scores and the top three images are awarded ribbons.

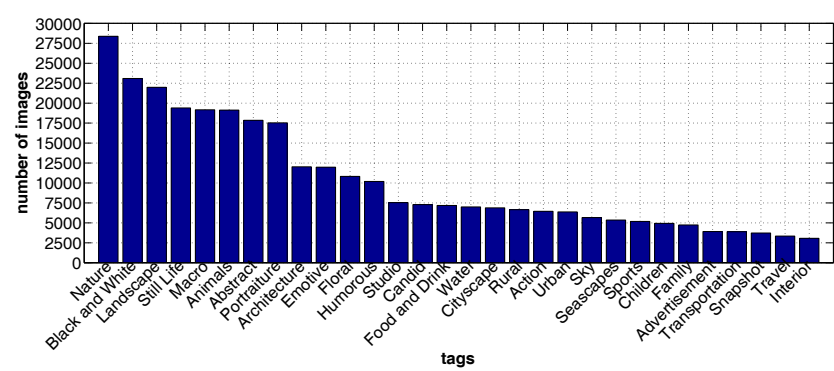

Fig. 5 Frequency of the 30 most common semantic tags in AVA. The tags cover a wide range of content and styles. The most popular content-related tags are nature and landscape, while the most popular styles are black and white and macro.

achieved by manipulating camera configurations (such as shutter speed, exposure, or ISO level). We manually selected 72 Challenges corresponding to photographic styles and we identified three broad categories according to a popular photography manual Kodak 1982): Light, Color, Composition. We then merged similar challenges (e.g. "Duotones" and "Black \& White") and we associated each style with one category. The 14 resulting photographic styles along with the number of associated images are: Complementary Colors (949), Duotones (1,301), High Dynamic Range (396), Image Grain (840), Light on White (1,199), Long Exposure (845), Macro (1,698), Motion Blur (609), Negative Image (959), Rule of Thirds (1,031), Shallow DOF (710), Silhouettes $(1,389)$, Soft Focus $(1,479)$, Vanishing Point (674).

In the next two sections we focus on the key AVA annotations necessary for our goal of learning aesthetic attributes, namely the score distributions and textual comments. 


\subsection{Aesthetic preference as real-valued scores}

Annotations of aesthetic preference are typically in the form of real-valued scores. When multiple scores are given to an image, as it is the case with images derived from social networking sites like www. dpchallenge.com, a score distribution is formed. In this section, we analyze the rich score distributions (consisting on average of approximately 200 scores) available in AVA in order to gain a deeper understanding of such distributions and of what kind of information can be deduced from them.

Score distributions are largely Gaussian. Table 2 shows a comparison of Goodness-of-Fit (GoF), as measured by RMSE, between top performing distributions we used to model the score distributions of AVA. One sees that Gaussian functions perform adequately for images with mean scores between 2 and 8, which constitute $99.77 \%$ of all the images in the dataset. In fact, the RMSEs for Gaussian models are rarely higher than 0.06. This is illustrated in Fig. 6. Each plot shows a density function obtained by averaging the score distributions of images whose mean score lies within a specified range. The averaged score distributions are usually well approximated by Gaussian functions (see Figures 6(b) and 6(c) . We also fitted Gaussian Mixture Models with three Gaussians to the distributions but we only found minor improvement with respect to one Gaussian. Beta, Weibull and Generalized Extreme Value distributions were also fitted to the score distributions, but gave poor RMSE results.

Non-Gaussian distributions tend to be highly-skewed. This skew can be attributed to a floor and ceiling effect (Cramer and Howitt 2004), occurring at the low and high extremes of the score scale. This can be observed in Figures 6(a) and 6(d). Images with positivelyskewed distributions are better modeled by a Gamma distribution $\Gamma(s)$, which may also model negativelyskewed distributions using the transformation $\Gamma^{\prime}(s)=$ $\Gamma\left(\left(s_{\min }+s_{\max }\right)-s\right)$, where $s_{\min }$ and $s_{\max }$ are the minimum and maximum scores of the score scale.

Standard Deviation is a function of mean score. Box-plots of the variance of scores for images with mean scores within a specified range are shown in Fig. 7. It can be seen that images with "average" scores (scores around 4,5 and 6) tend to have a lower variance than images with scores greater than 6.6 or less than 4.5. Indeed, the closer the mean score gets to the extreme scores of 1 or 10 , the higher the probability of a greater variance in the scores. This is likely due to the nonGaussian nature of score distributions at the extremes of the score scale.

\begin{tabular}{lccc}
\hline Mean score & \multicolumn{3}{c}{ Average RMSE } \\
& Gaussian & $\Gamma$ & $\Gamma^{\prime}$ \\
\hline $1-2$ & 0.1138 & $\mathbf{0 . 0 7 1 7}$ & 0.1249 \\
\hline $2-3$ & 0.0579 & $\mathbf{0 . 0 4 6 0}$ & 0.0633 \\
$3-4$ & $\mathbf{0 . 0 2 7 9}$ & 0.0444 & 0.0325 \\
$4-5$ & $\mathbf{0 . 0 2 9 1}$ & 0.0412 & 0.0389 \\
$5-6$ & $\mathbf{0 . 0 2 8 8}$ & 0.0321 & 0.0445 \\
$6-7$ & 0.0260 & $\mathbf{0 . 0 2 5 0}$ & 0.0455 \\
$7-8$ & $\mathbf{0 . 0 2 6 8}$ & 0.0273 & 0.0424 \\
$8-9$ & 0.0532 & 0.0591 & $\mathbf{0 . 0 4 0 3}$ \\
\hline Average RMSE & $\mathbf{0 . 0 2 8 4}$ & 0.0335 & 0.0429 \\
\hline
\end{tabular}

Table 2 Goodness-of-Fit per distribution with respect to mean score: the last row shows the average RMSE for all images in the dataset. The Gaussian distribution was the best-performing model for $62 \%$ of images in AVA.

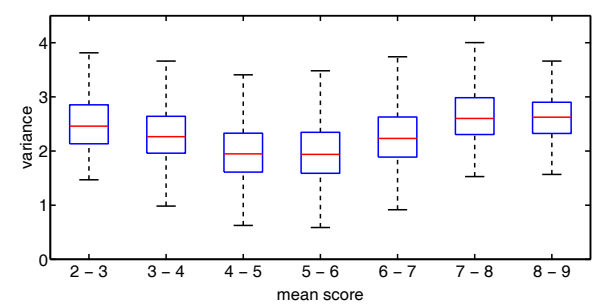

Fig. 7 Distributions of variances of score distributions, for images with different mean scores. The variance tends to increase with the distance between the mean score and the midpoint of the score scale.

\section{Images with high variance are often non-conven-} tional. To gain an understanding of the additional information a distribution of scores may provide, we performed a qualitative inspection of images with low and high variance. Table 3 displays our findings. The styles and photographic techniques employed to shoot seem to correlate with the mean score photographs receive. For a given mean value however, images with a high variance seem more likely to be edgy or subject to interpretation, while images with a low variance tend to use conventional styles or depict conventional subject matter. This is consistent with our intuition that an innovative application of photographic techniques and/or a creative interpretation of a challenge description is more likely to result in a divergence of opinion among voters. Examples of images with low and high score variances are shown in Fig. 8. The bottom-left photo in particular, submitted to the challenge "Faceless", had an average score of 5.46 but a very high variance of 5.27 . The comments it received indicate that while many voters found the photo humorous, others may have found it rude.

\section{Semantic content and aesthetic preference}

We evaluated aggregated statistics for each challenge using the score distributions of the images that were 


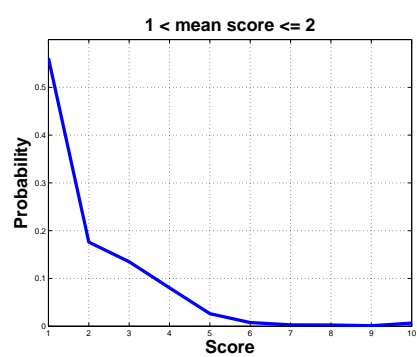

(a)

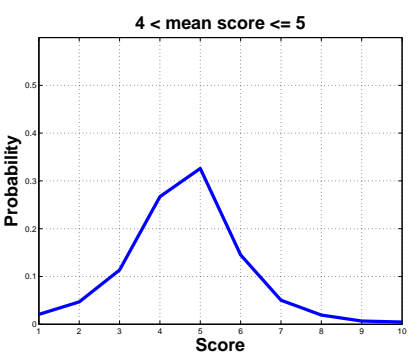

(b)

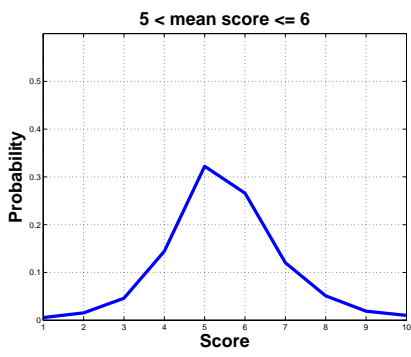

(c)

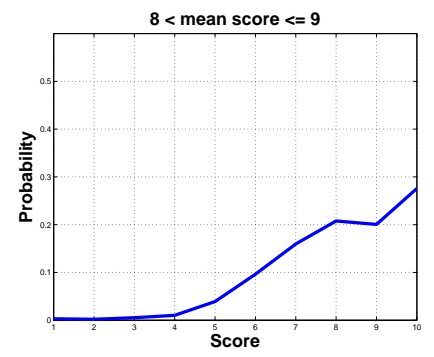

(d)

Fig. 6 Averaged distributions for images with different mean scores. Distributions with mean scores close to the mid-point of the score scale tend to be Gaussian, with highly-skewed distributions appearing at the end-points of the scale.

\begin{tabular}{|c|c|c|c|c|}
\hline & \multicolumn{3}{|c|}{ variance } \\
\hline & & low & & high \\
\hline \multirow[t]{2}{*}{ mean } & low & $\begin{array}{l}\text { poor, } \\
\text { tional } \\
\text { and/or } \\
\text { matter }\end{array}$ & $\begin{array}{r}\text { conven- } \\
\text { technique } \\
\text { subject }\end{array}$ & $\begin{array}{l}\text { poor, } \\
\text { conventional } \\
\text { nique and/or } \\
\text { matter }\end{array}$ \\
\hline & high & $\begin{array}{l}\text { good, } \\
\text { tional } \\
\text { and/or } \\
\text { matter }\end{array}$ & $\begin{array}{r}\text { conven- } \\
\text { technique } \\
\text { subject }\end{array}$ & $\begin{array}{l}\text { good, } \\
\text { conventional } \\
\text { nique and/or subject } \\
\text { matter }\end{array}$ \\
\hline
\end{tabular}

Table 3 By qualitatively inspecting images with different means and variances, we identified 4 categories of images with shared patterns, common quality features and subjects.

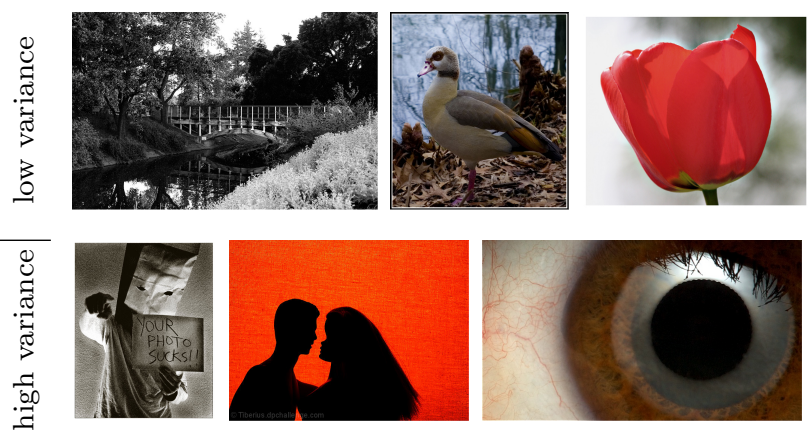

Fig. 8 Examples of images with mean scores around 5 but with different score variances. High-variance images have nonconventional styles or subjects.

submitted. Fig. 9 shows a histogram of the mean score of all challenges. As expected, the mean scores are approximately normally distributed around the mid-point of the score scale. We inspected the titles and associated descriptions of the challenges at the two extremes of this distribution. We did not observe any semantic coherence between the challenges in the right-most part of the distribution. However, it is worth noticing that two "masters' studies" (where only members who have won awards in previous challenges are allowed to participate) were among the top 5 scoring challenges. We use the arousal-valence emotional plane

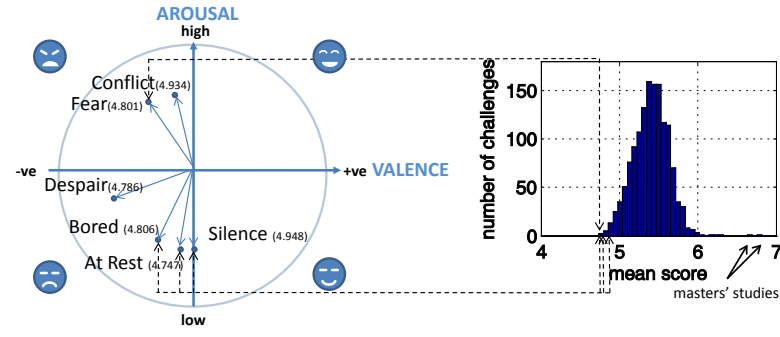

Fig. 9 Challenges with a lower-than-normal average vote are often in the left quadrants of the arousal-valence plane. The two outliers on the right are masters' studies challenges.

(Russell 1980) to plot the challenges on the left of the distribution (the low-scoring tail). The dimension of valence ranges from highly positive to highly negative, whereas the dimension of arousal ranges from passive to active. In particular, among the lowestscoring challenges we identified: \#1 "At Rest" (av. vote $=4.747), \# 2$ "Despair" (av. vote=4.786), \#3 "Fear" (av.vote=4.801), \#4 "Bored" (av. vote=4.8060), \# 6 "Pain" (av. vote=4.818), \#23 "Conflict" (av. vote $=4.934), \# 25$ "Silence" (av. vote= 4.948), \#30 "Shadows" (av. vote= 4.953), \#32 "Waiting" (av. vote.=4.953), \#39 "Obsolete" (av.vote= 4.9740). In each case, the photographers were instructed to depict or interpret the emotion or concept of the challenge's title. This suggests that themes in the left quadrants of the arousal-valence plane (see Fig. 9h bias the aesthetic judgments towards lower scores.

We investigated the relationship between the title and description of a challenge and the mean of the variance of the score distributions of images submitted to that challenge. We found that the majority of free study challenges were among the bottom 100 challenges by variance, with 11 free studies among the bottom 20 challenges. Free study challenges have no restrictions or requirements as to the subject matter of the submitted photographs. The low variance of these types of challenges suggests that challenges with specific re- 
quirements tend to lead to a greater variance of opinion, probably with respect to how well entries adhere to these requirements.

\subsection{Aesthetic preference as textual comments}

Of the 255,530 images in AVA, most of them $(253,903)$ received at least one comment from a member of the social network. There are two phases in which comments may be given. In the first phase, the challenge is ongoing and the comments and votes given to images are not yet visible to the community. In this phase, a user is allowed to give a comment to an image after giving that image a score. Comments given in this phase should therefore be unbiased with respect to the opinions of other members. In the second phase, the challenge has been completed and the results are public. Comments given in this phase are therefore likely to be biased in at least two ways. First, images which performed well during the challenge are likely to have a greater number of comments as they are more visible, being high in the rankings for that challenge. Second, the comments given to an image in this period may be influenced by the results of the challenge and the comments it has already received.

The guidelines for commenting ${ }^{1}$ encourage the users to leave comments when voting and to include advice for improving the work. As such, comments typically express the member's opinion on the quality of the photograph, their justifications for giving a certain score, as well as critiques of the strengths and weaknesses of the photograph. For example, the top right image in Fig. 8 received the following comment:

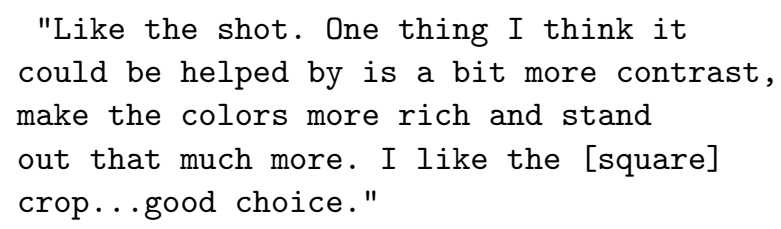

These comments are a rich source of information about the reasons for which an individual may assign a particular aesthetic score to an image.

We investigated several properties of the comments given to images in AVA: the number of available comments; the commentators' activity; and the quality of available comments.

Number of comments: Statistics on the number and length of comments given to images are shown in Table 4. On average, an image tends to have about 11 comments, with a comment having about 18 words on

\footnotetext{
1 http://www.dpchallenge.com/help_faq.php\#howcomments
}

average. However, the mean number of comments given during a challenge is greater than the mean number of comments given after. Interestingly, the length of comments given during a challenge is on average much shorter than those given after the challenge. Our observations lead us to believe that this is due to a "critique club" effect. The critique club comprises volunteer members who give a detailed critique of images which they have been assigned to review. The website states that ${ }^{2}$

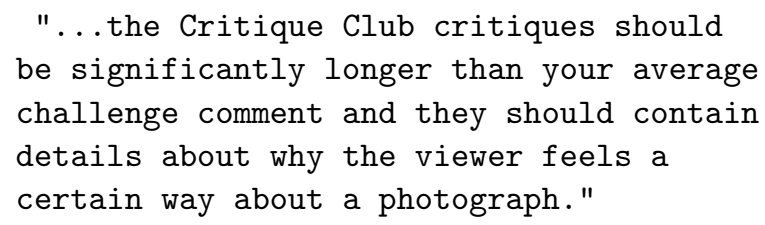

For an image to be critiqued, its author must request a critique when submitting the image. These critiques are then posted to the image's page after voting has finished. As such comments are detailed and long, they likely increase the average length of comments given after challenge completion.

As shown in Table 5, the number of comments made about an image varies significantly with respect to the mean score given to that image. Unsurprisingly, highscoring images have a large number of comments compared to other images. This bias is more pronounced when comparing the number of comments given during voting to the number of comments given after. Images with mean scores close to the midpoint of the score scale tend to have very few comments, perhaps because it is difficult to form an opinion about an image that is neither clearly bad nor clearly good. However, the mean length of the comments given to such images is much higher than the global average. This may be because critique club comments are often one of the few comments given to such images, and bias the mean length towards a higher number.

Commentators' activity: For the images in AVA, 27,557 unique members made 2,934,728 comments. Fig. 10 shows the commenting activity of these commentators. We found that approximately $86 \%$ of users write comments only occasionally, while the remaining 3,983 users are regular commentators who have authored at least 100 comments.

Technical content in comments: We investigated the words present in comments to determine how many comments contained technical content related to photographic techniques and aesthetic quality. We manually selected the technical words found among the 1,000

\footnotetext{
2 http://www.dpchallenge.com/forum.php?action=read
} \&FORUM_THREAD_ID $=19842$ 


\begin{tabular}{cccc}
\hline Statistics & During challenge & After challenge & Overall \\
\hline comments per image $(\mu$ and $\sigma)$ & $9.99(8.41)$ & $1.49(4.77)$ & $11.49(11.12)$ \\
words per comment $(\mu$ and $\sigma)$ & $16.10(8.24)$ & $43.51(61.74)$ & $18.12(11.55)$ \\
\hline
\end{tabular}

Table 4 Statistics on comments in AVA. On average, an image tends to have about 11 comments, with a comment having about 18 words on average. As the statistics in columns 2 and 3 attest however, commenting behavior is quite different during and after challenges.

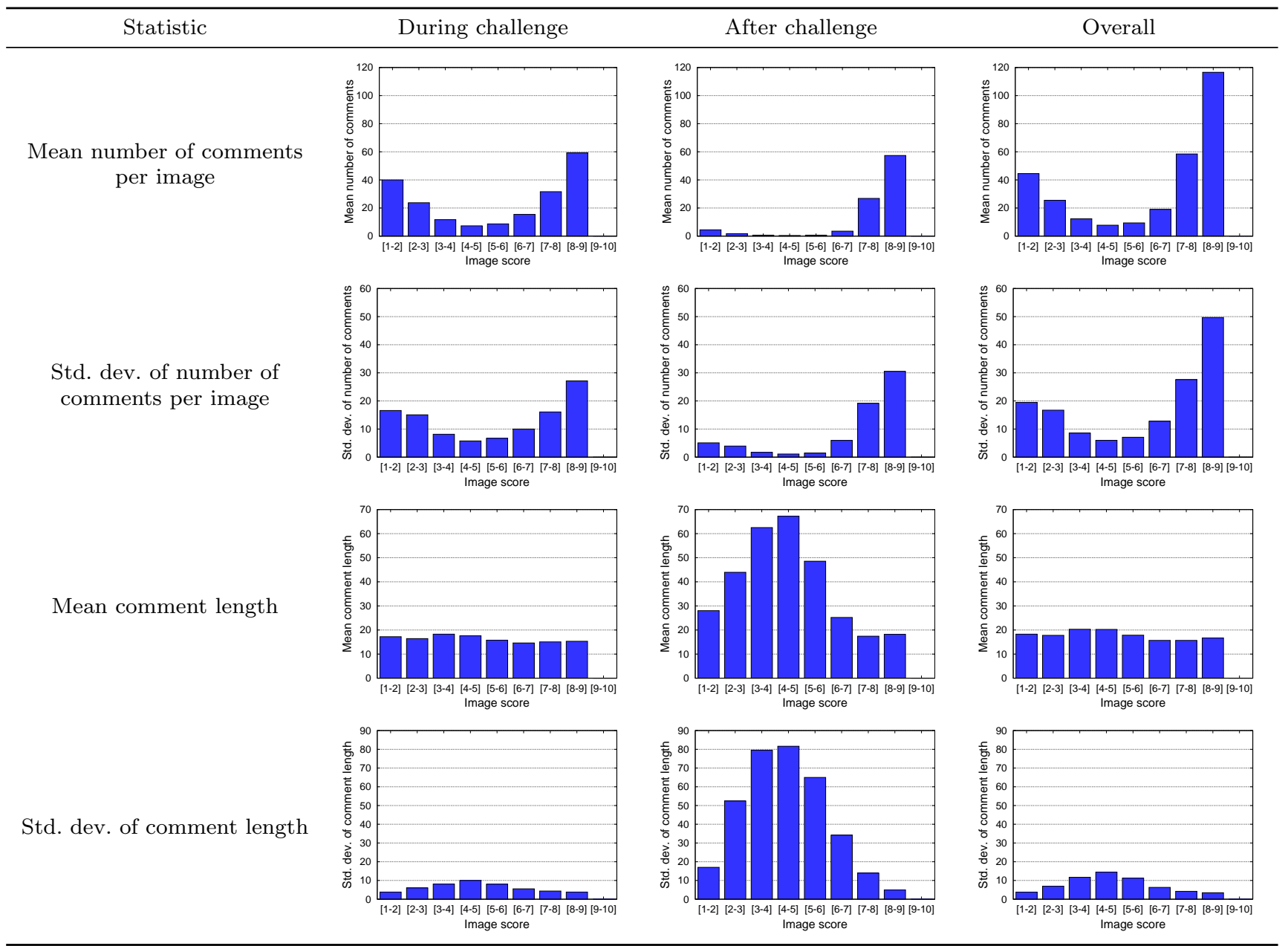

Table 5 Number of comments in the AVA database and their length (in number of words) for images within the given score range. More and longer comments are made during challenges than afterwards. Overall, high-scoring images have a large number of comments compared to other images.

most frequently used words in the set of comments. We found 149 such words, examples of which are "exposure", "lighting", "vivid" and "texture". We note that this was a non-exhaustive list of the technical terms included in the corpus of comments. Even so, we found that $77 \%$ of comments include at least one of these technical words, and among these comments, 2.8 words were used on average.

We next describe how AVA's textual comments, used in conjunction with its real-valued scores can be leveraged to automatically discover visual attributes.

\section{Discovering textual attributes}

As stated earlier, we aim to use the user comments of the AVA dataset as a textual resource, since they contain very rich information about aesthetics. However, such comments are quite noisy: they can be very short as shown in the previous section and they are written in a very spontaneous manner. This makes our task particularly challenging.

In this section we first describe how the comments found in AVA can be used to obtain textual features for image aesthetics (section 4.1). We then describe in sec- 


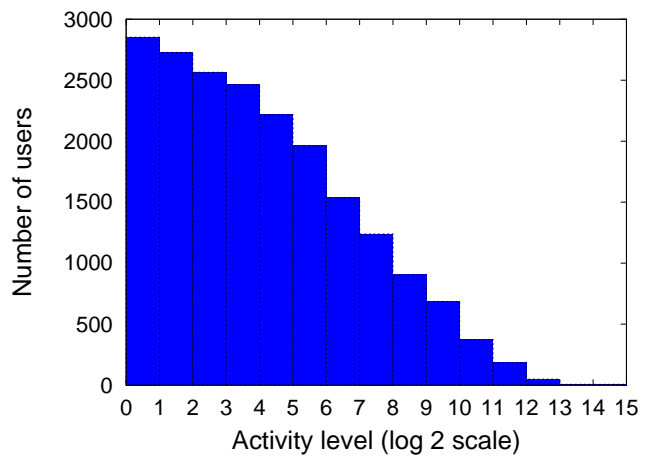

Fig. 10 Histogram of number of users for different activity levels, where activity level is denoted by number of comments made. The activity level ranges from 1 to 24,232 comments.

tion 4.2.1 a first approach to attribute discovery which is fully unsupervised as it only relies on comments. We show its limitations and then propose in section 4.2.2 a supervised approach to attribute discovery which relies on the user scores.

\subsection{Textual features for image aesthetics}

Textual information has only recently been used to infer the aesthetics of images. (Geng et al 2011) created several bags-of-textual-words from different text sources related to web images. These sources included the image url and the title of the page on which the image is found. The textual vocabulary consisted of the 8 words in their dataset with the most information gain. (San Pedro et al 2012) used a sentiment analysis method to extract features from textual comments given to images by users. These features were the 49 most frequents words used in comments to refer to visual characteristics of images. Examples include "color", "composition" and "lighting".

As mentioned previously, the textual comments in AVA contain detailed opinions of users on the aesthetic properties of images. We used these comments to create descriptors comprised of term frequency-inverse document frequency (tf-idf) weights. Such descriptors have been very successful in information retrieval applications (Joachims 1998).

We first created a tokenized corpus using the comments of all images in AVA. The terms in the corpus which are repeated at least 10 times are used to create a vocabulary. We merge all the critiques related to an image into a single textual document. Merging the generally very short and noisy comments averages noise and thus leads to a more robust representation. We tokenize and spell-check each document and we remove stop-words and numbers. Each document is represented as a bag-of-words (BOW) histogram using the term frequency-inverse document frequency weighting (tf-idf). Hence, each commented image is associated with a bag-of-words vector.

We constructed vocabularies comprising: (i) unigrams or single word terms; (ii) bigrams or terms compromising two words that appear consecutively in a comment; or (iii) unigrams and bigrams. We chose to investigate these particular vocabulary compositions as they achieved good performance in the text categorization literature (Bekkerman and Allan 2004).

Our unigram, bigram and unigram+bigram vocabularies contained 30,595, 138,993 and 169,560 terms respectively. Bigrams retain some of the semantic relations between words, while this is completely lost in the case of unigrams. On the other hand, unigrams which are highly informative of aesthetic impressions are not present in the bigram feature representation.

We evaluate our TF-IDF vectors using a subset of AVA which we will call sAVA. This subset of 70,000 images was created by (San Pedro et al 2012) for evaluating textual features derived from user comments. We randomly select from sAVA 30,000 images for training, 10,000 for validation, and 30,000 images for testing. To evaluate on an aesthetics classification we must derive binary labels from the user scores. To do this, we follow (Datta et al 2008) and set two thresholds $\theta_{1}=5+\delta / 2$ and $\theta_{2}=5-\delta / 2$. We then annotate each image with the label "beautiful" if $q_{a v}(i) \geq \theta_{1}$ and "bad" if $q_{a v}(i) \leq \theta_{2}$. $\delta$ is used to artificially create a gap between high and low quality images, as pictures lying in this gap are likely to represent noisy data in the peer-score process. As in (Datta et al 2006) we vary this $\delta$ value in our experiments. Increasing the value $\delta$ obviously makes the classification task easier. Note that images belonging to the "bad" class are not necessarily bad per se. They only correspond to images that received lower scores.

Results are shown in Fig. 11. We found that vectors constructed from a unigram vocabulary performed better than those formed from a bigram vocabulary, while vectors formed from a vocabulary of unigrams and bigrams out-performed both, findings which are consistent with text categorization problems (Bekkerman and Allan 2004). However, the gain in performance was modest and unlikely to justify the increase in training time and storage requirements due to the increased vocabulary size.

We also measured the correlation between our classifier scores and the scores of the test images. As shown in Table 6, our textual features out-perform the text and visual-based features of (San Pedro et al 2012), and the state-of-the-art visual features of (Marchesotti et al 2011). This shows that our textual features can be used to predict attractiveness, thus validating their 


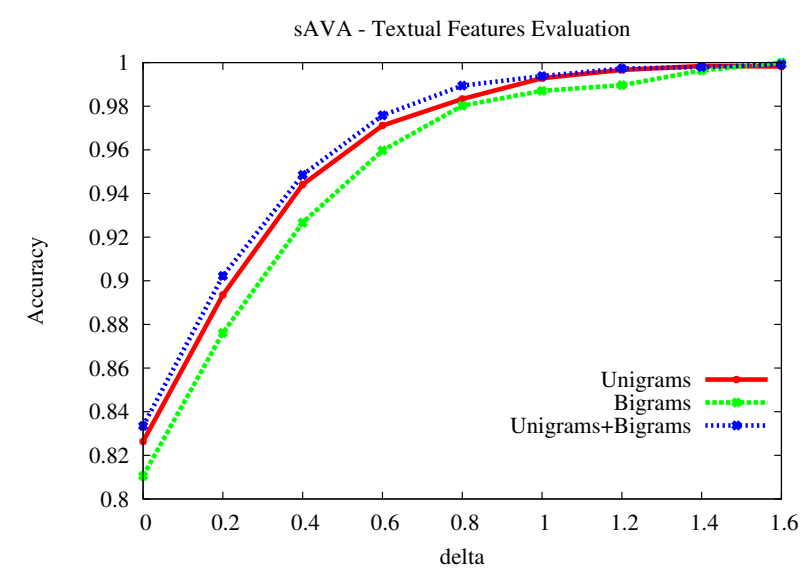

Fig. 11 Classification accuracy on sAVA using different flavors of textual descriptors. Unigrams+Bigrams outperform Unigrams which outperform Bigrams.

\begin{tabular}{lc}
\hline Method & Spearman's $\rho$ \\
\hline San Pedro et al., visual-based & 0.3133 \\
Marchesotti et al., visual-based & 0.4524 \\
San Pedro et al., comment-based & 0.5839 \\
San Pedro et al., visual+comment-based & 0.6107 \\
Unigrams & 0.8335 \\
Bigrams & 0.8209 \\
Unigrams + Bigrams & $\mathbf{0 . 8 4 3 3}$ \\
\hline
\end{tabular}

Table 6 Regression performance on sAVA dataset. Our proposed textual features outperform the state-of-the-art feature extraction schemes.

usefulness for our task. We next describe how we automatically discover attributes from these features.

\subsection{Attributes discovery from textual features}

We aim to use the features, or terms, in our textual vocabulary as aesthetic attributes. We next present two approaches to this task: one without and one with supervisory data.

\subsubsection{Unsupervised attributes discovery}

As a first attempt to discover attributes, we use the unsupervised probabilistic Latent Semantic Analysis (pLSA) (Hofmann 2001) algorithm on the BOW histograms. The hope is that the learned topics correlate with photographic techniques and therefore they are interpretable as attributes. In Table 7, we report some of the most interpretable topics discovered by pLSA with $K=50$ hidden topics. We can see that some topics relate to general appreciation and mood (T3, T11, T28, T20), to photographic techniques and colors (T35, T27, T49) or to semantic labels (T8, T14, T37). Despite the relevance of these topics to visual attractiveness, we cannot directly use them as attributes: they are too vague (i.e. not granular enough) and much manual postprocessing would be needed to extract something useful. Experiments with different numbers of topics $K$ did not lead to more convincing results.

\subsubsection{Supervised attributes discovery}

We devise an alternative strategy based on the following approach: we use the attractiveness scores as supervisory information to mitigate the noise of textual labels. The hope is that by using attractiveness scores we will be able to identify interpretable textual features that are highly correlated with aesthetic preference and use them to predict aesthetic scores.

Selecting discriminative textual features. We mine beautiful and ugly attributes by discovering which terms can predict the aesthetic score of an image.

For this purpose, we train an elastic net (Zou and Hastie 2005) support vector regressor to predict aesthetic scores and, at the same time, select textual features. It is a regularized regression method that combines an $\ell_{2}$-norm and a sparsity-inducing $\ell_{1}$-norm. Let $N$ be the number of textual documents. Let $D$ be the dimensionality of the BOW histograms. Let $\mathbf{X}$ be the $N \times D$ matrix of documents. Let $y$ be the $N \times 1$ vector of scores of aesthetic preference (the score of an image is the average of the scores it received). Our goal if to learn a $D$-dimensional vector $\hat{\beta}$ that reflects the contribution of each BOW entry to the aethetic preference. Toward this purpose, we optimize the following objective:

$\hat{\beta}=\arg \min _{\beta}\|\mathbf{y}-\mathbf{X} \beta\|^{2}+\lambda_{1}\|\beta\|_{1}+\lambda_{2}\|\beta\|^{2}$

where $\lambda_{1}$ and $\lambda_{2}$ are the regularization parameters.

We first experiment with the same vocabulary of $D \approx 30,000$ unigrams described in section 4.1 . We crossvalidated the regularization parameters using Spearman's $\rho$ correlation coefficient and we selected the values of $\lambda_{1}$ and $\lambda_{2}$ providing highest performances with 1,500 non-zero $\beta$ coefficients. We analyze the candidate attributes by sorting them according to $|\beta|$ (see Table 8 ) to verify their interpretability. By inspecting the most discriminant unigrams, we can see that the ones at the top of each rank relate to specific visual attributes (e.g. grainy, blurry). But others can be ambiguous (e.g. not, doesn't, poor) and interpreting them is rather problematic.

To resolve these ambiguities we turn to bigrams. As mentioned in section 4.1, bigrams preserve some of the semantic relations between neighboring words, 
T3: $\quad$ ribbon, congrats, congratulations, deserved, first, red, well, awesome, yellow, great, glad, fantastic, excellent, page, wonderful, happy

T11: beautiful, wow, amazing, congratulations, top, congrats, finish, love, stunning, great, wonderful, excellent, awesome, perfect, fantastic, gorgeous, absolutely, capture

T28: idea, creative, clever, concept, cool, executed, execution, original, well, great, pencil, job, creativity, thought, top, work, shannon, interesting, good

T20: funny, lol, laugh, hilarious, humor, expression, haha, title, fun, made, oh,love,smile, hahaha, great

T35: motion, panning, blur, speed, movement, shutter, moving, blurred, abstract, blurry, effect, pan, stopped, sense, camera, fast, train, slow, background, exposure

T27: colors, red, colours, green, abstract, color, yellow, orange, beautiful, colour, border, vibrant, complementary, composition, leaf, lovely, love, background, bright, purple

T49: selective, desat, desaturation, red, use, color, works, processing, desaturated, saturation, editing, fan

T8: portrait, eyes, face, expression, beautiful, skin, hair, character, portraits, eye, smile, nose, lovely, self, girl, look, wonderful, great, lighting, crop

T14: cat, cats, kitty, eyes, fur, pet

T37: sign, road, signs, street, stop

Table 7 Sample topics generated by pLSA for $K=50$ topics.

\begin{tabular}{|c|c|}
\hline Unigrams & $\begin{array}{l}\text {-great }(0.4351) \text {, like }(0.3301) \text {, excellent }(0.2943) \text {, love }(0.2911) \text {, beautiful }(0.2704) \text {, done }(0.2609) \text {, very }(0.2515) \text {, } \\
\text { well }(0.2465) \text {, shot }(0.2228) \text {, congratulations }(0.2223) \text {, perfect }(0.2142) \text {, congrats }(0.2114) \text {, wonderful }(0.2099) \text {, } \\
\text { nice }(0.1984) \text {, wow }(0.1942) \text {, one }(0.1664) \text {, top }(0.1651) \text {, good }(0.1639) \text {, awesome }(0.1636) \text {, }\end{array}$ \\
\hline Unigrams & $\begin{array}{l}\text { sorry }(-0.2767) \text {, focus }(-0.2345) \text {, blurry }(-0.2066) \text {, small }(-0.1950) \text {, not }(-0.1947) \text {, don }(-0.1881) \text {, doesn }(-0.1651) \text {, } \\
\text { flash }(-0.1326) \text {, snapshot }(-0.1292) \text {, too }(-0.1263) \text {, grainy }(-0.1176) \text {, meet }(-0.1122) \text {, out }(-0.1054) \text {, try }(-0.1041) \text {, } \\
\text { low }(-0.1013) \text {, poor }(-0.0978) \text {, distracting }(-0.0724) \text {, }\end{array}$ \\
\hline Bigrams+ & $\begin{array}{l}\text { well done }(0.6198) \text {, very nice }(0.6073) \text {, great shot }(0.5706) \text {, very good }(0.3479) \text {, great job }(0.3287) \text {, your top } \\
(0.3262) \text {, my favorites }(0.3207) \text {, top quality }(0.3198) \text {, great capture }(0.3051) \text {, lovely composition }(0.3014) \text {, my } \\
\text { top }(0.2942) \text {, nice shot }(0.2360) \text {, th placing }(0.2330) \text {, great lighting }(0.2302) \text {, great color }(0.2245) \text {, excellent } \\
\text { shot }(0.2221) \text {, good work }(0.2218) \text {, well executed }(0.2069) \text {, great composition }(0.2047) \text {, my only }(0.2032)\end{array}$ \\
\hline Bigrams- & $\begin{array}{l}\text { too small }(-0.3447) \text {, too blurry }(-0.3237) \text {, not very }(-0.3007) \text {, does not }(-0.2917) \text {, not meet }(-0.2697) \text {, wrong } \\
\text { challenge }(-0.2561) \text {, better focus }(-0.2280) \text {, not really }(-0.2279) \text {, sorry but }(-0.2106) \text {, really see }(-0.2103) \text {, poor } \\
\text { focus }(-0.2068) \text {, too out }(-0.2055) \text {, keep trying }(-0.2026) \text {, see any }(-0.2021) \text {, not sure }(-0.2017) \text {, too dark } \\
(-0.2007) \text {, next time }(-0.1865) \text {, missing something }(-0.1862) \text {, just don }(-0.1857) \text {, not seeing }(-0.1785)\end{array}$ \\
\hline
\end{tabular}

Table 8 Most discriminant unigrams and bigrams with their regression coefficient $\beta$. Bigrams are in general more interpretable than unigrams since they can capture the polarity of comments and critiques.

which is essential for our purpose of obtaining humaninterpretable attributes. In particular, bigrams capture non-compositional meanings that a simpler feature does not (Riloff et al 2006). For instance the word "lighting" does not have an intrinsic polarity while a bigram composed of "great" and "lighting" can successfully clarify the meaning. As such, the use of bigrams is a popular choice in opinion mining (Pang et al 2012).

We performed regression on the 90,000 most frequent bigrams among those described in section 4.1. using the same procedure employed for unigrams. The bottom rows of Table 8 show the bigrams which receive the highest/lowest regression weights. As expected, regression weights implicitly select those features as the most discriminant ones for predicting attractiveness. The highest weights correspond to "beautiful" attributes while the lowest weights correspond to "ugly" attributes. It is noteworthy that we use an Elastic Net to overcome the limitations of other sparsity-inducing norms like LASSO (Tibshirani 1996) in the feature selection tasks: if there is a group of features among which the pairwise correlations are very high, then the LASSO tends to select only one random feature from the group (Zou and Hastie 2005). In our case, LASSO produces a compact vocabulary of uncorrelated attribute labels, but also a very small number of labeled images. This is problematic because we need as many annotated images as possible at a later stage to train one visual classifiers for each attribute.

Clustering bigrams. The effect of the Elastic Net on correlated features can be seen by looking at table 8; as expected, the Elastic Net tolerates correlated features (synonym bigrams) such "well done" or "very nice", "beautiful colors" and "great colors". This augments the number of annotated images, but it requires us to handle synonyms in the vocabulary of attributes. For this reason, we compact the list of 3,000 candidate bigrams (1,500 for Beautiful attributes and 1,500 for Ugly attributes) with Spectral Clustering (Ng et al 2002). We cluster the beautiful and ugly bigrams separately. We heuristically set the number of clusters to 200 (100 Beautiful and 100 Ugly clusters) and we create the similarity matrices with a simple but very effective measure of bigram similarity: we calculate the Levenshtein dis- 
tance among the second term within each bigram and we discard the first term. This approach is based on the following intuition: most bigrams are composed of a first term which indicates the polarity and a second term which describes the visual attribute e.g. "lovely composition", "too dark", "poor focus". What we obtain is an almost duplicate-free set of attributes, and a richer set of images associated with them. Some sample clusters are reported here below:

C18: ['beautiful', 'colors'] ['great', 'colors'] ['great', 'colours'] ['nice', 'colors']

C56: ['challenge', 'perfectly'] ['just', 'perfect']

C67: ['nicely', 'captured'] ['well', 'captured'] ['you', 'captured'] C89: ['excellent', 'detail'] ['great', 'detail'] ['nice', 'detail']). We randomly draw a bigram from each cluster to name the corresponding attribute.

\section{Learning visual attributes}

The goal is now to learn one visual attribute model for each discovered textual attribute. However, it is difficult to hand-design a different visual model for each of our 200 attributes. Therefore we propose to learn such attribute models from generic visual features, in the same manner that Marchesotti et al (2011) proposed to use generic visual features to learn preference models. In this section, we first describe the chosen generic visual features that we use to represent our images. We then explain how attribute models are learned and then reranked based on visualness.

\subsection{Visual features for image aesthetics}

We extract 128-dim SIFT (Lowe 1999) and 96-dim color descriptors (Clinchant et al 2007) from 24x24 patches on dense grids every 4 pixels at 5 scales. We reduce dimensionality by using a 64-dim PCA. These low-level descriptors are aggregated into an image-level signature using the Fisher Vector (FV) (Chatfield et al 2011). We use visual vocabularies of 64 Gaussians and encode some rough image layout information by concatenating FVs extracted from the whole image, its 4 quadrants and three equally-sized horizontal image strips. We chose this image representation as it has been shown to result in state-of-the-art performance for semantic (Chatfield et al 2011) as well as aesthetic tasks (Marchesotti et al 2011). We compute one SIFT-based and one color-based representation per image and we concatenate them. This leads to a combined 131,072-dim representation which is PQ-compressed (Jégou et al 2011) to reduce the memory footprint and to enable all images to be kept in RAM.

\subsection{Attribute learning from visual features}

A categorization problem is considered to be large-scale if either (i) the size of the feature space; (ii) the number of classes; or (iii) the number of training samples is large. Given the high dimensionality of the FVs, the large number of images available in AVA (approx. $250,000)$, and the large number of attribute classifiers to be learned, our classification problem resides squarely in the large-scale paradigm. It is therefore fundamental to employ a scalable solution.

For this reason, we use linear classifiers optimized with an online learning algorithm, namely Stochastic Gradient Descent (SGD) (Bottou and Bousquet 2007). We use a regularized logistic regression objective function. Using logistic loss (rather than a hinge loss for instance) provides a probabilistic interpretation of the classification scores, which is a desirable property since we are training attributes. The resultant linear classifiers are our visual attribute models. In the previous section, we enforced interpretability and discriminability of the attribute labels using attractiveness scores as a supervision mechanism. However, this choice does not ensure that all these attributes can be recognized by a computer. This is the reason why we measure "visualness" using the Area Under the ROC Curve (AUC) calculated for each individual attribute. In particular, we benchmark the classification performances of each attribute (1-vs-all) and we rank them using AUC. We show the top 50 attributes in Figure 12 for Ugly and Beautiful attributes. Our first observation is that beautiful attributes perform better than ugly attributes do. This is not surprising since the latter attributes were trained with fewer images: as shown in Table 5, people are less likely to comment on low-quality images, limiting the training set for ugly attributes. Second, we notice that attributes which detect lighting conditions and colors (e.g. too dark, great colour, too harsh) perform better than more complex visual concepts such as interesting idea, bit distracting, very dramatic.

It is also worth noting that both SIFT and colorbased features are useful for the classification of attributes. This is not surprising since some attributes are very color-related ("nice colors", "black background"), while others are well-captured by gradient information ("leading lines", "great sharpness"). As Fig. 13 shows, combining SIFT and color features results in increased performance. We also compared the performances of two learning approaches: 1-vs-rest against multi-class classifiers (Crammer and Singer 2002). As shown in Fig. 14, the former strategy provided significantly better results experimentally. This may result from a large overlap between attribute classes in feature space, a 

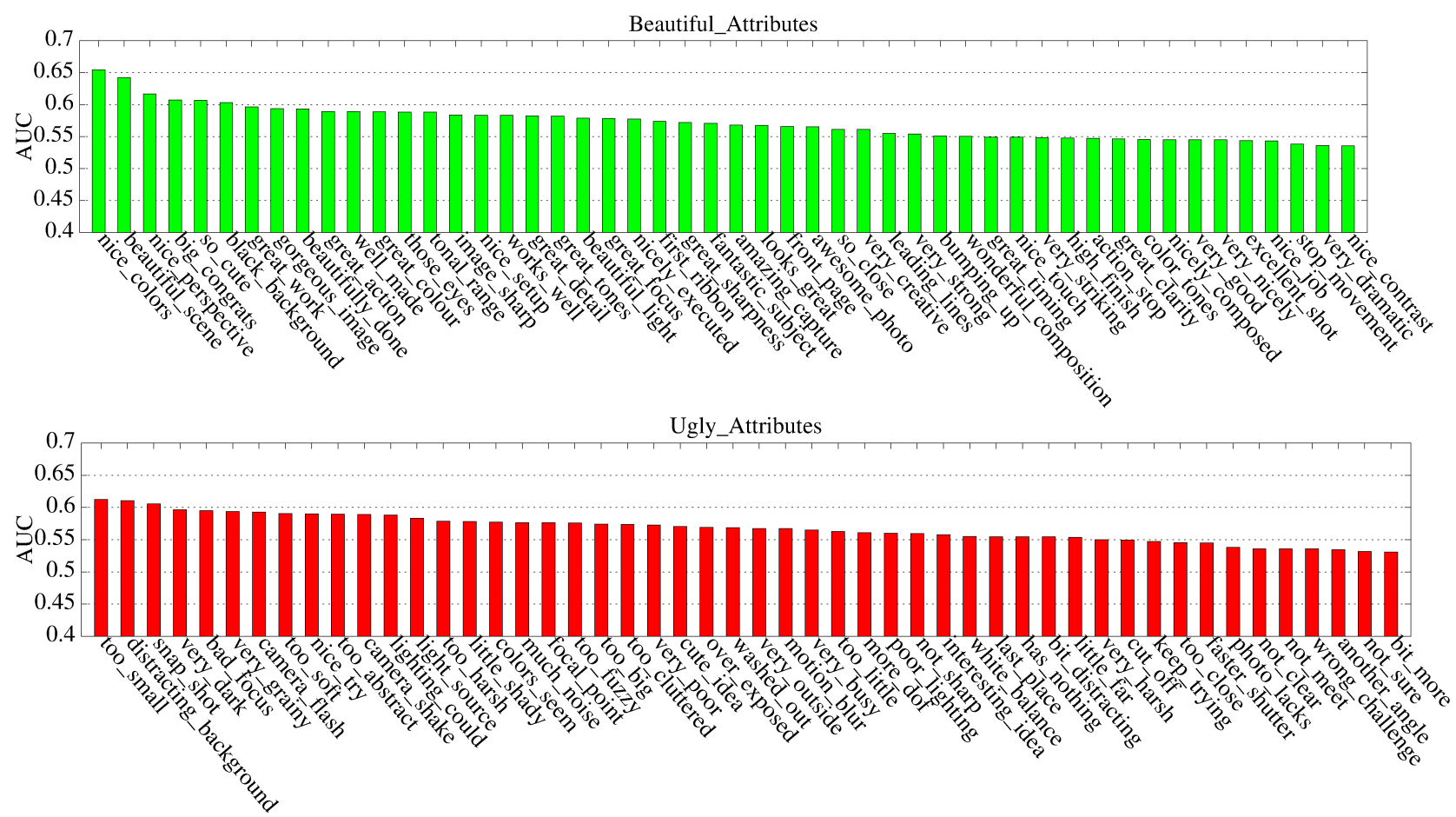

Fig. 12 Area Under the Curve (AUC) calculated for the top 50 Beautiful and Ugly attributes.

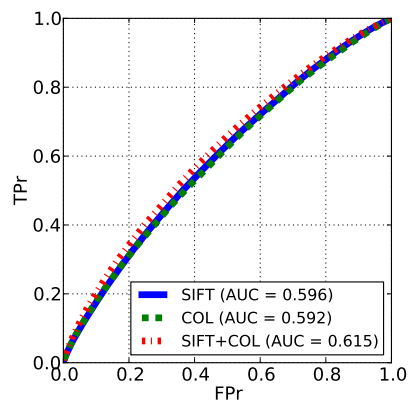

(a) Beautiful Attributes

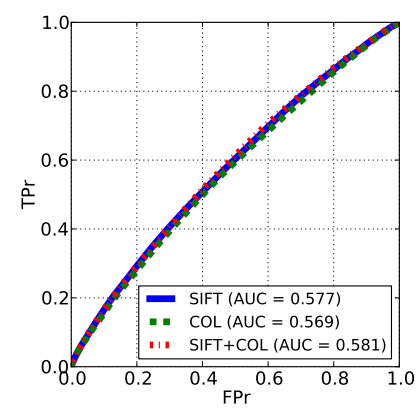

(b) Ugly Attributes

Fig. 13 ROC curves for SIFT features and color statistics features, averaged over (a) beautiful; and (b) ugly attributes.

regime in which multi-class classification has been observed to perform poorly compared to one-vs-rest classification (Akata et al 2014).

\subsection{The attribute representation}

To form an image representation using our learned attribute classifiers, we compute the classifier scores given to the image's FV by the 100 best-performing (in terms of AUC) beautiful and ugly attributes. This results in a 200-dimensional real-valued attribute vector which we can use to train preference models (see section 6 for several applications). Fig. 15 shows a random

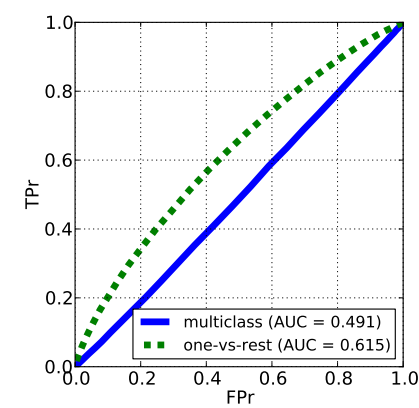

(a) Beautiful Attributes

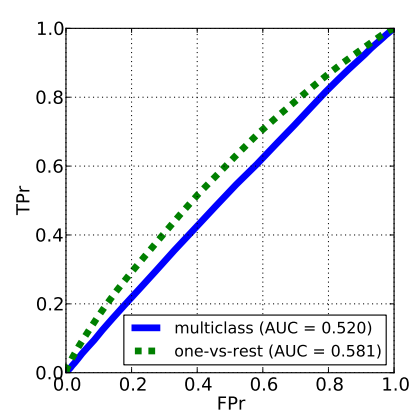

(b) Ugly Attributes
Fig. 14 ROC curves for multi-class classifiers and 1-vsrest classifiers, averaged over (a) beautiful; and (b) ugly attributes.

sample of images and their 5 nearest neighbors in the 200-dimensional attribute space, as well as the original high-dimensional FV space. The nearest neighbors often have similar color and composition attributes and, as with textual queries, similar semantic content. When the query image has strong stylistic or compositional attributes, this is reflected in its nearest neighbors in attribute space. This can be seen in the first query image in Fig. 15, whose strong sepia tones and uncluttered composition are reflected in its nearest nearest neighbors in the attribute space. The last query image contains strong line patterns and a black and white palette, attributes which are well represented in 

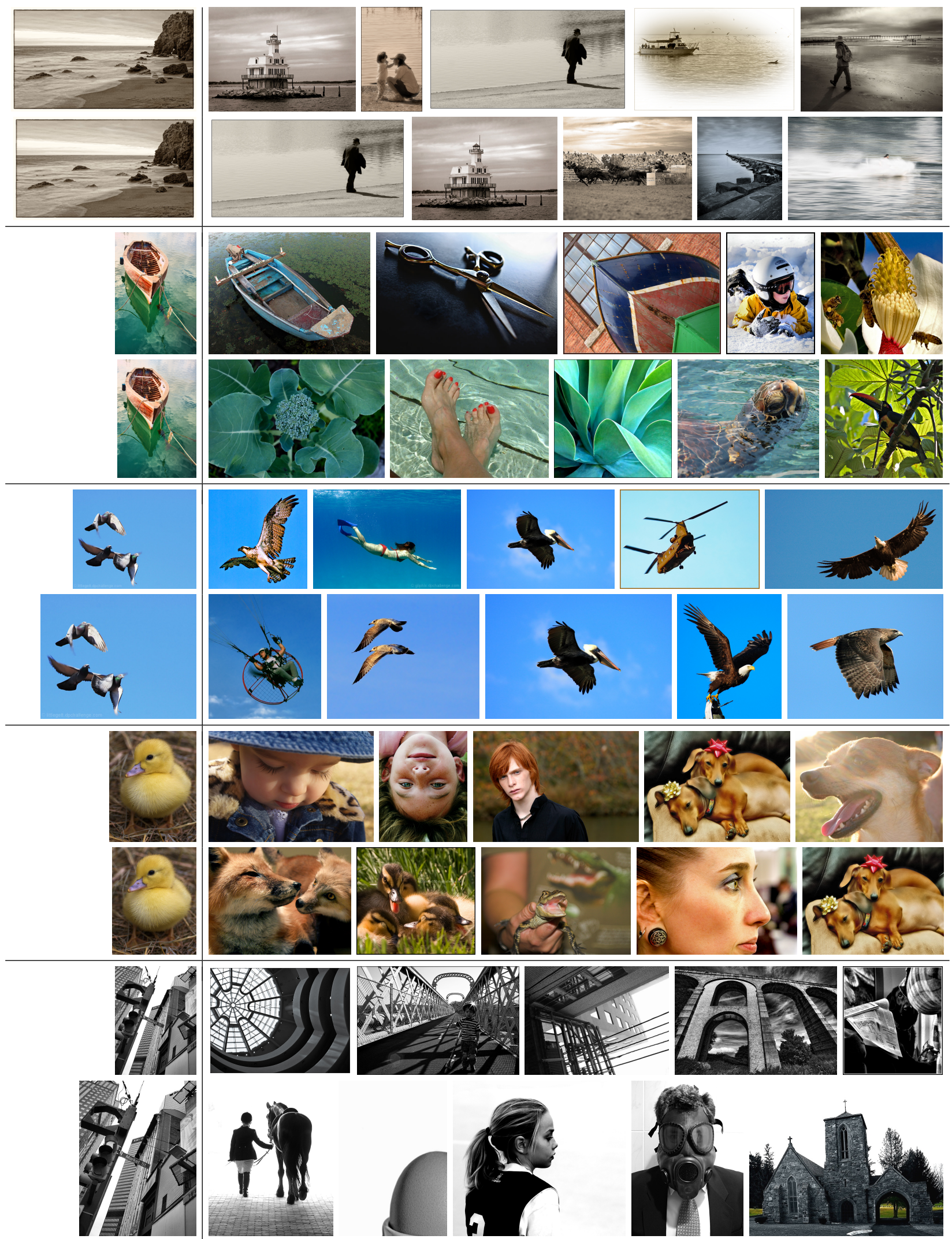

Fig. 15 Five randomly-chosen images and their 5 nearest neighbors in (i) the 200-dimensional attribute space (top rows); (ii) the original high-dimensional FV space (bottom rows). 
its nearest neighbors. The nearest neighbors in the FV space reflect these attributes less uniformly.

\section{Applications}

In this section we consider three applications of the proposed attributes: aesthetic prediction, image tagging, and query-by-text image retrieval.

\subsection{Aesthetic prediction}

In some cases, we might be interested in giving a binary answer regarding the attractiveness of an image: beautiful versus ugly. Such binary decisions are the organizing principle behind online photo-sharing venues such as www.imgur.com (via "like" and "dislike" buttons) and http://www.reddit.com/r/itookapicture/ (via "upvote" and "downvote" buttons). We therefore propose to use our learned attributes to make such a prediction and compare to the approach of (Marchesotti et al 2011) which is based on generic image features and is to date the best-performing baseline on AVA dataset. To make the comparison with (Marchesotti et al 2011), we use the same FV features and linear classifiers in both cases. As can be seen in Fig. 16(a), attributes perform on par with low-level generic features, despite the significant difference in dimensionality (131,072 dimensions for the low-level features and 200 dimensions for the attributes). Therefore attributes achieve equivalent performance $(\mathrm{AUC}=0.718$ for attributes, versus 0.715 for generic generic features) and introduce the possibility of replacing a single image attractiveness label (beautiful or ugly) with the labels of the most responsive attributes. Note that while one can also reduce the dimensionality of the FVs using random projections or PCA, there is no guarantee that the new dimensions will be human-interpretable, and even if so, they would need to be manually labeled.

Generalization performance. To investigate the generalizability of the attributes, we evaluated their performance on images obtained from Photo.net. We downloaded a random selection of $10 \mathrm{~K}$ training and testing images, and $7 \mathrm{~K}$ validation images, along with their mean aesthetic scores. Our attribute vectors achieved $\mathrm{AUC}=0.631$ on the test set, compared to $\mathrm{AUC}=0.659$ for generic FV (see also Fig. 16(b)], demonstrating that our attributes can indeed be applied to predict aesthetic preference for images collected in an entirely different context. In addition, for the price of a small performance decrease compared to $\mathrm{FV}$, one gains interpretability of aesthetic preference, without using

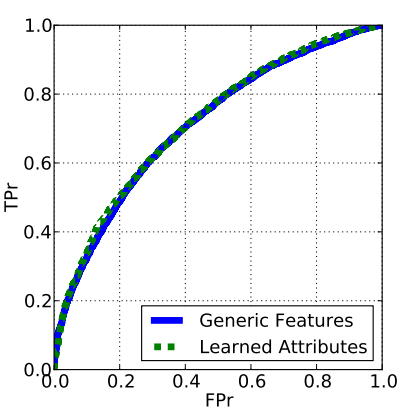

(a) AVA

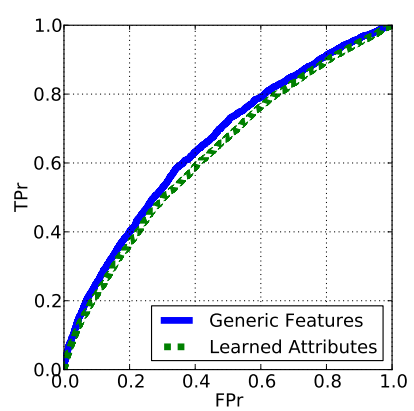

(b) Photo.net
Fig. 16 Aesthetic preference prediction: comparison between learned attributes and generic visual features (Marchesotti et al 2011) for the AVA dataset (a), and with generalization to Photo.net images (b).

any textual meta-data that may be associated with the Photo.net images. We note that this scenario, in which images are assumed to have no aesthetics-related textual meta-data, is by far the most typical in existing image corpora.

\subsection{Image tagging}

We now go beyond tagging an image as beautiful or ugly, as a binary decision can be too aggressive for a problem as subjective as aesthetic quality. Indeed, it could form a positive or negative prior in the user's mind in contradiction to his/her tastes and opinions.

To gain users' consensus we design an application that not only predicts aesthetic quality (Is this image beautiful or ugly?) but also produces a qualitative description of the aesthetic properties of an image in terms of beautiful/ugly attributes. As can be seen from the examples of Table 9 , this strategy gives the user higher degree of interpretation of the aesthetic quality. For instance, while many users might agree that the leftmost image is a beautiful picture, others might disagree that the yellow flower on the right is ugly: in general people tend to refuse criticism. Instead, with attributes such as more light, more depth field of view and not sure the application takes a more cautious approach and enables the user to form his/her own opinion. Finally, we realize that these are just plausible hypotheses that should be tested with a full-fledged user study. However such an evaluation is out of the scope of this work.

\subsection{Query-by-text image retrieval}

We now show how the learned attributes, evaluated quantitatively in section 5.2 (see Fig. 12), can be used 


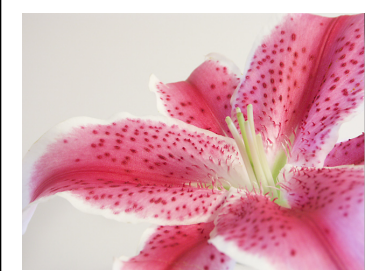

great_macro, very_pretty, great_focus, nice_detail, so_cute

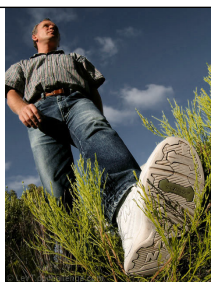

great_capture, great_angle, nice_perspective, lovely_photo, nice_detail

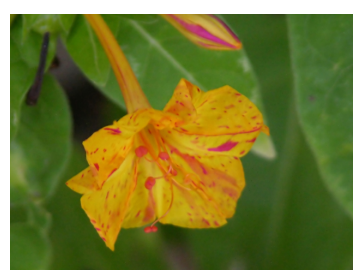

soft_focus, not_sure, more_dof, too_busy, blown_out more_light,

sharper_focus, more_dof

Table 9 Sample results for an image annotation application where the aesthetic quality of each image is described using the 5 most reactive attributes.

to perform attribute-based image retrieval. We display the top-returned results of several queries for Beautiful and Ugly attributes in the mosaic of Fig. 17. We notice that the images clearly explain the labels discovered in AVA even for fairly complex attributes such as too busy, blown out, white balance (note the various kind of color casts present in the images of row 6) or Much noise in the last row.

The top-ranked images sometime contain very similar semantic content. For example, the top-ranked images for the attribute nice perspective are almost all images of architectural structures. This indicates that our visual attributes may be highly correlated with semantic information, which is unsurprising given that photographic style is very content-dependent. An interesting topic for future work would involve leveraging semantic annotations (which are present in AVA) in order to design learning strategies that overcome this potential limitation.

Our learned attributes may also be used in combination with semantic models to enable joint attribute/semantic queries. To demonstrate this we train classifiers, using the same train/val/test splits, for the 8 semantic categories studied by Murray et al (2012a): "animal", "architecture", "cityscape", "floral", "fooddrink", "landscape", "portrait", and "stilllife". For a joint query such as landscape with great colors, we first apply the landscape semantic classifier and the great colours attribute classifier to the test images. These scores are converted to probabilities, multiplied and then sorted to produce a final ranking of the test images with respect to the joint query. We use multiplication to approximate the "AND" operator, as we want images relevant to both terms in the query to be the most highly ranked. While more sophisticated fusions are possible (Murray et al 2012b), their evaluation for this task falls out of the score of this work. Fig. 18 shows the top 5 results for some sample joint queries. Once again, the images clearly reflect the attributes, and also contain relevant semantic content. Note for instance that the two landscape-related queries return very different top results due to the different attributes requested: $d r a-$ matic sky vs great colours.

\section{User Study}

Images in AVA are only partially annotated with semantic and attribute information. For instance, although many of the displayed results shown in Fig. 18 are reasonable, they could be counted as errors because they lack the corresponding semantic or aesthetic tag. Consequently, a quantitative evaluation of the image retrieval results that relies solely on AVA annotations would provide a very pessimistic performance estimate.

Therefore, to assess the quality of these results we performed a user study using CrowdFlower ${ }^{3}$ one of the leading crowdsourcing platforms. The setup of the experiment was the following: we showed crowdsourcing workers an image and we asked two questions about its relevance to the query (e.g. "1. Determine if the image subject is ARCHITECTURE", "2. Determine if the image features the photographic technique LEADING LINES"). The semantic and attribute relevance were assessed independently for two reasons: firstly, we wanted to simplify as much as possible the task of the workers. Secondly, we wanted to compare the performance of semantic to aesthetic attribute retrieval. A three-value scale (Agree, Unsure, Disagree) was chosen. Three judgments per image were sufficient to get a high degree of agreement among randomly-chosen workers $(>84 \%)$.

We launched the experiment on the 5 joint queries shown in Fig. 18. For each query the top 200 images retrieved by the automatic classifier were used in the study. Images were randomized before they were shown to workers.

\footnotetext{
3 http://www.crowdflower.com/
} 

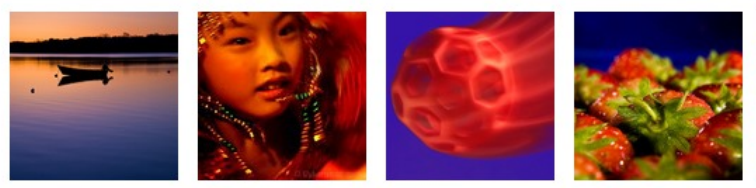

beautiful_colors
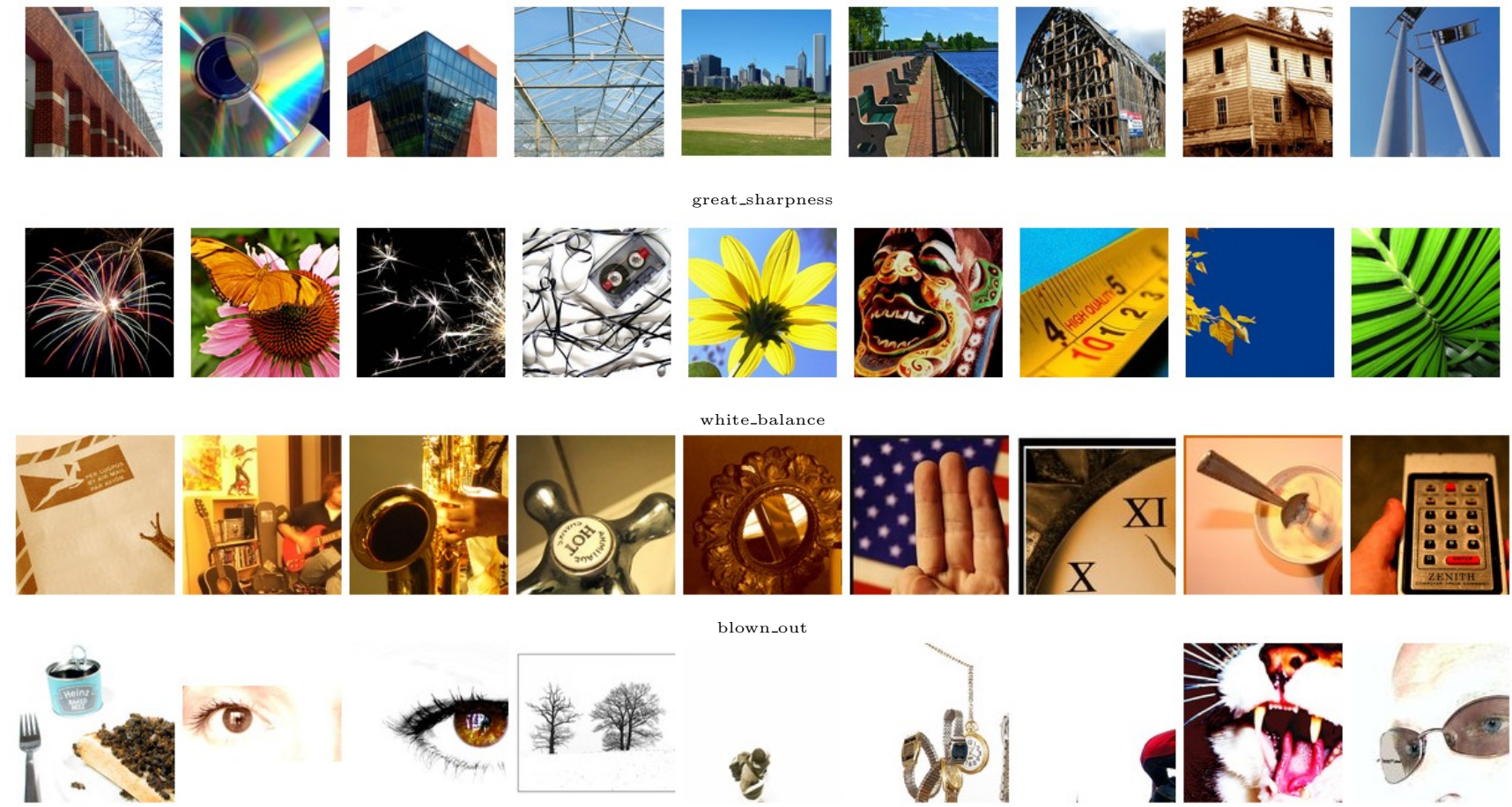

blown_out
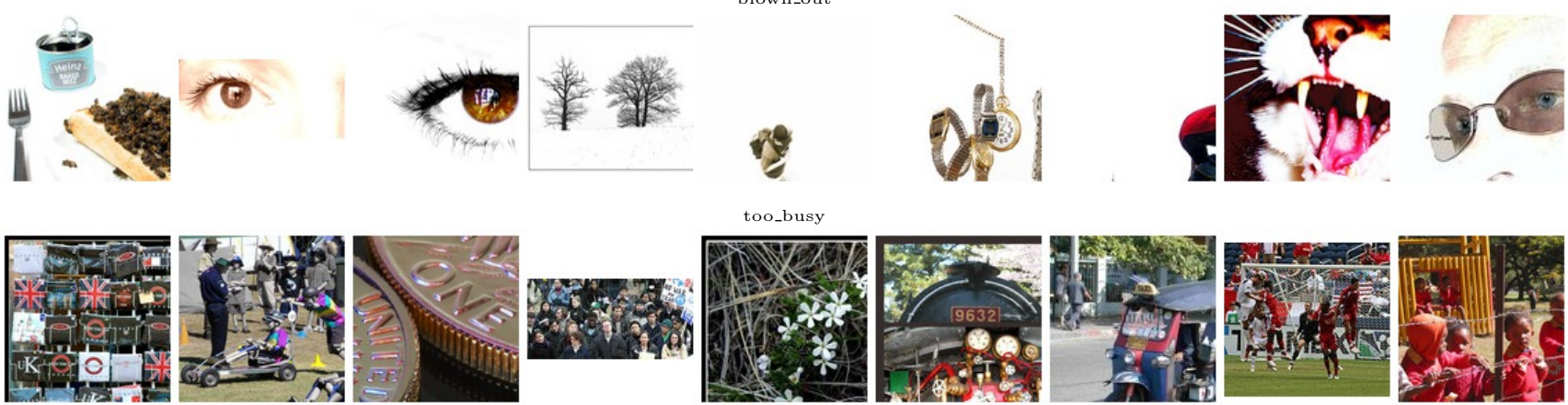

Fig. 17 Images with top scores for some representative beautiful and ugly attributes.

To assess the quality of the ranks we used Precision@K (see Fig. 19(a)]. To get maximum precision, both attributes had to be assessed by three workers in an image. The best performing queries are landscape/dramatic sky and animals/macro.

To gain a deeper understanding of these results, we also counted the errors among aesthetic and semantic attributes on a per-query basis. The results are shown in Fig. 19(b), Two conclusions can be drawn: firstly, most errors are semantic. Secondly, flowers and animals are the queries where the content attributes have lowest performance. While aesthetic attributes based on color ( e.g. "black background", "great colour") or simple composition properties such as macro ( big object and out of focus background) perform well, other more complex composition attributes such as leading lines are more difficult to capture.
We also measured the confidence of responses for each query, shown in Fig. 19(c), The confidence here is measured as the agreement between the responses of the three workers on a per-image basis. As can be seen, confidence on content attributes is higher than confidence on aesthetic attributes: this coincides with the fact that, in general, semantic attributes are less subjective than aesthetic attributes.

\section{Conclusions}

In this paper, we tackled the problem of visual attractiveness analysis using visual attributes as mid-level features. Despite the great deal of subjectivity of the problem, we showed that we can automatically learn semantically-meaningful attributes using the unique conjunction of image, scoring, and textual data in the 


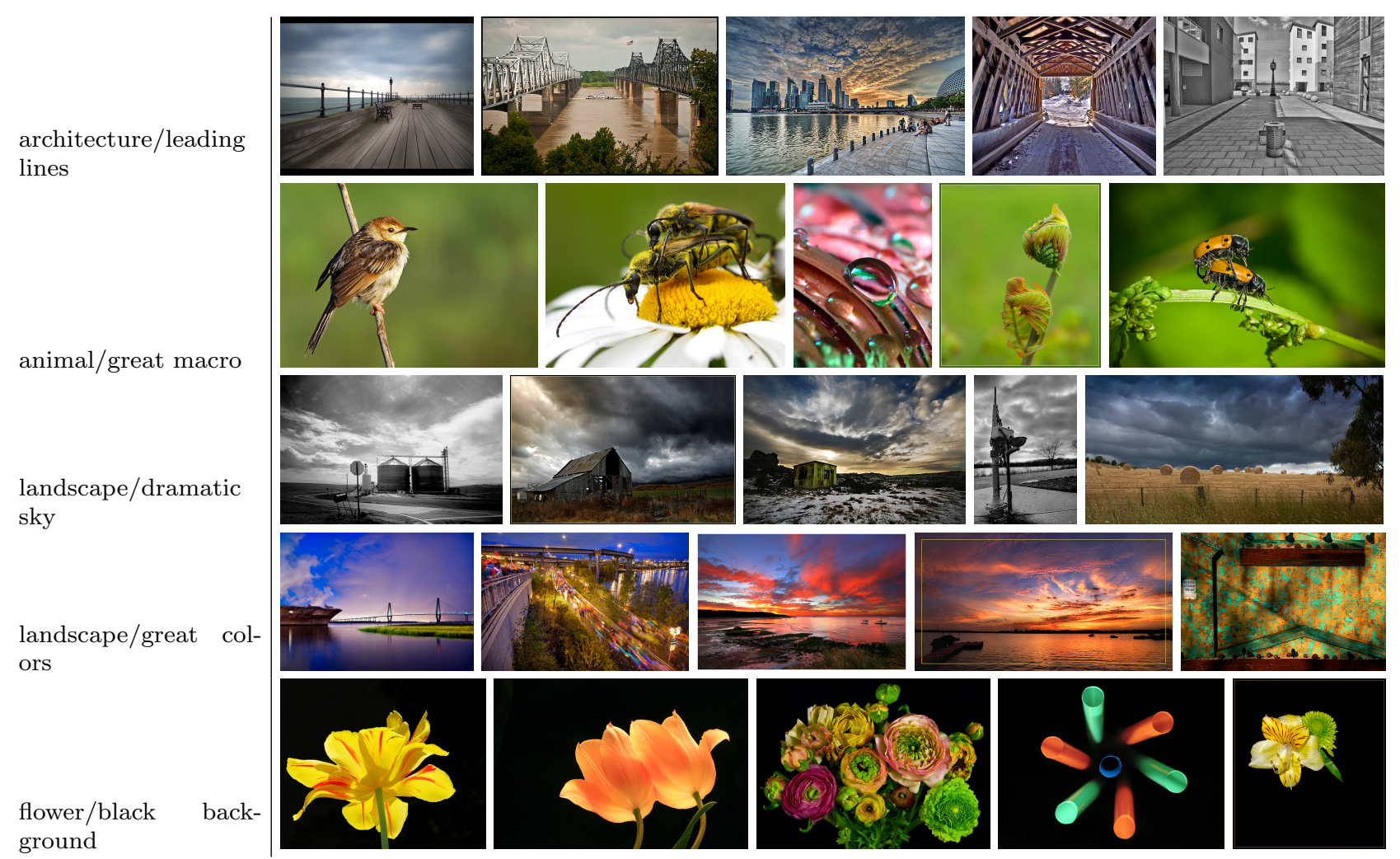

Fig. 18 Images with top scores for some sample joint attribute/semantic queries.

AVA dataset, for which we provided an in-depth analysis. We demonstrated the effectiveness of our attributes in various applications such as score prediction, image auto-tagging or image retrieval. Future work will focus on testing with users the advantage of our beautiful and ugly attributes and on mitigating biases introduced by semantic information.

Acknowledgements The authors would like to thank JeanMichel Renders for the discussions about text analysis and Isaac Alonso for having supported the experimental work of this paper.

\section{References}

"aesthetics" E (2012) The American Heritage ${ }^{\circledR}$ Dictionary of the English Language, Fourth Edition

Akata Z, Perronnin F, Harchaoui Z, Schmid C (2014) Good practice in large-scale learning for image classification. Pattern Analysis and Machine Intelligence, IEEE Transactions on 36(3):507-520

Bekkerman R, Allan J (2004) Using bigrams in text categorization. Technical Report IR-408 Department of Computer Science, University of Massachusetts, Amherst, MA

Berg AC, Berg TL, Daume H, Dodge J, Goyal A, Han X, Mensch A, Mitchell M, Sood A, Stratos K, et al (2012) Understanding and predicting importance in images. In: CVPR, pp 3562-3569

Berg T, Berg A, Shih J (2010) Automatic attribute discovery and characterization from noisy web data. ECCV
Bottou L, Bousquet O (2007) The tradeoffs of large scale learning. In: NIPS

Chatfield K, Lempitsky V, Vedaldi A, Zisserman A (2011) The devil is in the details: an evaluation of recent feature encoding methods. In: BMVC

Chatterjee A (2011) Neuroaesthetics: a coming of age story. Journal of Cognitive Neuroscience 23(1):53-62

Clinchant S, Csurka G, Perronnin F, Renders JM (2007) Xrce participation to ImageEval. In: ImageEval Workshop at CVIR

Cramer D, Howitt D (2004) The SAGE dictionary of statistics, 1st edn. SAGE, p. 21 (entry "ceiling effect"), p. 67 (entry "floor effect")

Crammer K, Singer Y (2002) On the algorithmic implementation of multiclass kernel-based vector machines. The Journal of Machine Learning Research 2:265-292

Csurka G, Dance C, Fan L, Willamowski J, Bray C (2004) Visual categorization with bags of keypoints. In: ECCV SLCV Workshop

Datta R, Wang JZ (2010) Acquine: aesthetic quality inference engine - real-time automatic rating of photo aesthetics. In: MIR

Datta R, Joshi D, Li J, Wang JZ (2006) Studying aesthetics in photographic images using a computational approach. In: ECCV

Datta R, Joshi D, Li J, Wang JZ (2008) Algorithmic inferencing of aesthetics and emotion in natural images: An exposition. In: ICIP

Dhar S, Ordonez V, Berg T (2011) High level describable attributes for predicting aesthetics and interestingness. In: CVPR

Donahue J, Grauman K (2011) Annotator rationales for visual recognition. In: ICCV 


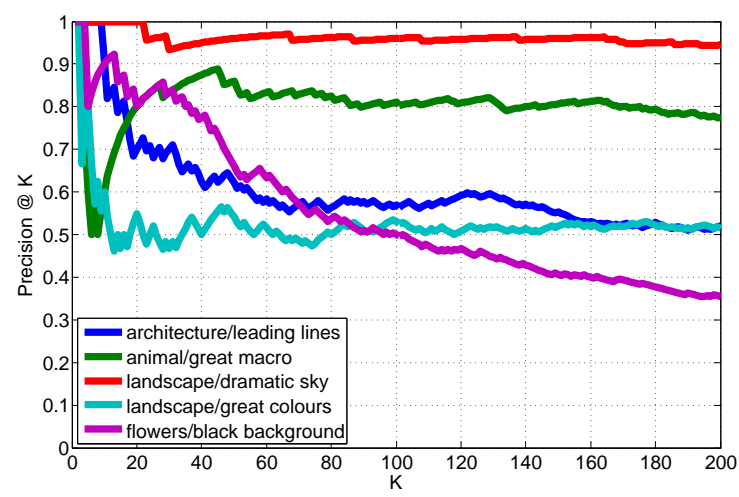

(a) Precision @ K

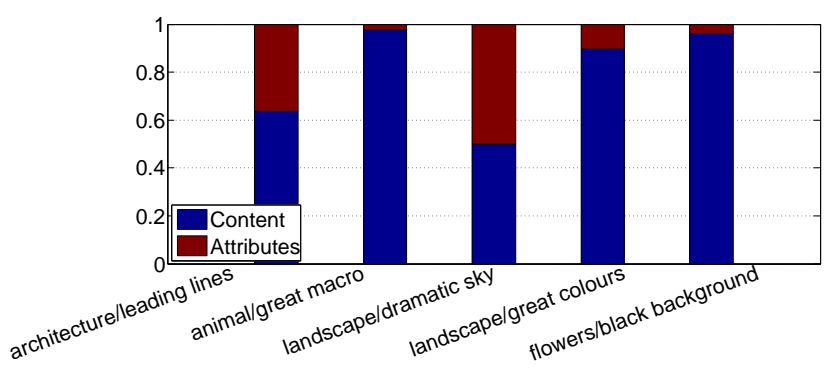

(b) Errors per query

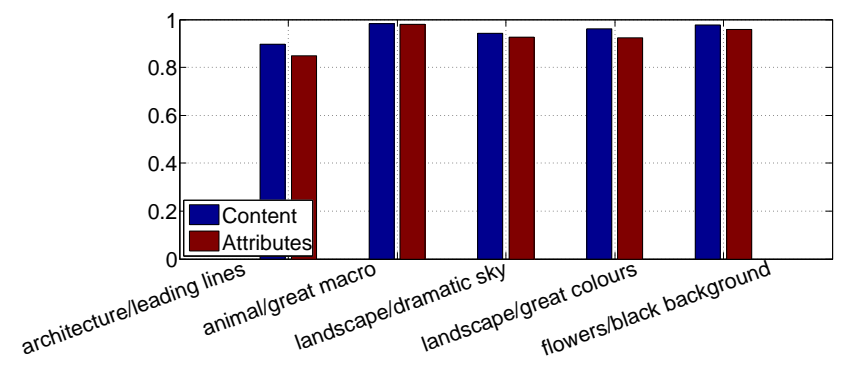

(c) Average annotation confidence

Fig. 19 Results of user study on image retrieval, showing (a) precision@K; (b) proportion of errors per query related to either semantics or attributes; and (c) the average annotation confidence, i.e. agreement, per query.

Duan K, Parikh D, Crandall D, Grauman K (2012) Discovering localized attributes for fine-grained recognition. In: CVPR

Farhadi A, Endres I, Hoiem D, Forsyth D (2009) Describing objects by their attributes. In: CVPR

Ferrari V, Zisserman A (2007) Learning visual attributes. In: NIPS

Geng B, Yang L, Xu C, Hua X, Li S (2011) The role of attractiveness in web image search. In: ACM-MM

Gracyk T (2011) Hume's aesthetics. In: Zalta EN (ed) The Stanford Encyclopedia of Philosophy, winter 2011 edn

Hammermeister K (2002) The German aesthetic tradition. Cambridge University Press

Hofmann T (2001) Unsupervised learning by probabilistic latent semantic analysis. Machine Learning
Isola P, Parikh D, Torralba A, Oliva A (2011) Understanding the intrinsic memorability of images. In: NIPS

Jacobson E, Ostwald W (1948) Color harmony manual. Container Corporation of America

Jégou H, Douze M, Schmid C (2011) Product quantization for nearest neighbor search. IEEE TPAMI

Joachims T (1998) Text categorization with support vector machines: Learning with many relevant features. In: ECML

Joshi D, Datta R, Fedorovskaya E, Luong Q, Wang J, Li J, Luo J (2011) Aesthetics and emotions in images. Signal Processing Magazine, IEEE 28(5):94-115

Ke Y, Tang X, Jing F (2006) The design of high-level features for photo quality assessment. In: CVPR

Kodak (1982) How to take good pictures : a photo guide. Random House Inc

Krages B (2005) Photography: the art of composition. Allworth Press

Lampert C, Nickisch H, Harmeling S (2009) Learning to detect unseen object classes by between-class attribute transfer. In: CVPR

Lazebnik S, Schmid C, Ponce J (2006) Beyond bags of features: Spatial pyramid matching for recognizing natural scene categories. In: CVPR

Leder H, Belke B, Oeberst A, Augustin D (2004) A model of aesthetic appreciation and aesthetic judgments. British Journal of Psychology 95(4):489-508

Li C, Loui AC, Chen T (2010) Towards aesthetics: a photo quality assessment and photo selection system. In: ACMMM

Lowe D (1999) Object recognition from local scale-invariant features. In: ICCV

Luo W, Wang X, Tang X (2011) Content-based photo quality assessment. In: ICCV

Luo Y, Tang X (2008) Photo and video quality evaluation: Focusing on the subject. In: ECCV

Machajdik J, Hanbury A (2010) Affective image classification using features inspired by psychology and art theory. In: ACM MM, New York, NY, USA

Marchesotti L, Perronnin F (2013) Learning beautiful (and ugly) attributes. In: BMVC

Marchesotti L, Perronnin F, Larlus D, Csurka G (2011) Assessing the aesthetic quality of photographs using generic image descriptors. In: ICCV

Müller H, Clough P, Deselaers T, Caputo B (2010) ImageCLEF: Experimental Evaluation in Visual Information Retrieval, vol 32. Springer

Murray N, Marchesotti L, Perronnin F (2012a) AVA: A largescale database for aesthetic visual analysis. In: CVPR

Murray N, Marchesotti L, Perronnin F (2012b) Learning to rank images using semantic and aesthetic labels. In: BMVC

Ng AY, Jordan MI, Weiss Y, et al (2002) On spectral clustering: Analysis and an algorithm. NIPS

Obrador P, Schmidt-Hackenberg L, Oliver N (2010) The role of image composition in image aesthetics. In: ICIP

Obrador P, Saad M, Suryanarayan P, Oliver N (2012) Towards category-based aesthetic models of photographs. Advances in Multimedia Modeling pp 63-76

Oliva A, Torralba A (2001) Modeling the shape of the scene: a holistic representation of the spatial envelope. IJCV

Orendovici R, Wang J (2010) Training data collection system for a learning-based photographic aesthetic quality inference engine. In: ACM-MM

Pang B, Lee L, Vaithyanathan S (2012) Thumbs up?: sentiment classification using machine learning techniques. 
In: Proceedings of the ACL-02 conference on Empirical methods in natural language processing

Parikh D, Grauman K (2011a) Interactively building a discriminative vocabulary of nameable attributes. In: CVPR

Parikh D, Grauman K (2011b) Relative attributes. In: ICCV

Perronnin F, Dance C (2007) Fisher kernels on visual vocabularies for image categorization. In: CVPR

Perronnin F, Sánchez J, Mensink T (2010) Improving the fisher kernel for large-scale image classification. In: ECCV

Riloff E, Patwardhan S, Wiebe J, et al (2006) Feature subsumption for opinion analysis. In: Proceedings of the 2006 Conference on Empirical Methods in Natural Language Processing

Rohrbach M, Stark M, Szarvas G, Gurevych I, Schiele B (2010) What helps where-and why? semantic relatedness for knowledge transfer. In: CVPR

Russell J (1980) A circumplex model of affect. Journal of personality and social psychology

San Pedro J, Yeh T, Oliver N (2012) Leveraging user comments for aesthetic aware image search reranking. In: WWW

Shelley J (2012a) 18th century british aesthetics. In: Zalta EN (ed) The Stanford Encyclopedia of Philosophy, summer 2012 edn

Shelley J (2012b) The concept of the aesthetic. In: Zalta EN (ed) The Stanford Encyclopedia of Philosophy, spring 2012 edn

Sivic J, Zisserman A (2003) Video Google: A text retrieval approach to object matching in videos. In: ICCV

Tibshirani R (1996) Regression shrinkage and selection via the lasso. Journal of the Royal Statistical Society Series B (Methodological)

Wang J, Markert K, Everingham M (2009) Learning models for object recognition from natural language descriptions. In: BMVC

Yanai K, Barnard K (2005) Image region entropy: a measure of visualness of web images associated with one concept. In: ACM-MM

Yao L, Suryanarayan P, Qiao M, Wang J, Li J (2012) Oscar: On-site composition and aesthetics feedback through exemplars for photographers. IJCV

Zou H, Hastie T (2005) Regularization and variable selection via the elastic net. Journal of the Royal Statistical Society 